\title{
Anti-GD2 CAR-NKT cells in patients with relapsed or refractory neuroblastoma: an interim analysis
}

\author{
Andras Heczey $₫ 1,2,3 \bowtie$, Amy N. Courtney', Antonino Montalbano ${ }^{4}$, Simon Robinson', \\ Ka Liu ${ }^{1}$ ', Mingmei Li', Nisha Ghatwai', Olga Dakhova3', Bin Liu', Tali Raveh-Sadka", \\ Cynthia N. Chauvin-Fleurence ${ }^{\circ}{ }^{1}$, Xin Xu ${ }^{1}{ }^{1}$, Ho Ngai', Erica J. Di Pierro', Barbara Savoldo5, \\ Gianpietro Dotti $\odot^{5}$ and Leonid S. Metelitsa ${ }^{1,2,3 凶}$
}

\begin{abstract}
Vo24-invariant natural killer T (NKT) cells have shown potent anti-tumor properties in murine tumor models and have been linked to favorable outcomes in patients with cancer. However, low numbers of these cells in humans have hindered their clinical applications. Here we report interim results from all three patients enrolled on dose level 1 in a phase 1 dose-escalation trial of autologous NKT cells engineered to co-express a GD2-specific chimeric antigen receptor (CAR) with interleukin-15 in children with relapsed or resistant neuroblastoma (NCT03294954). Primary and secondary objectives were to assess safety and anti-tumor responses, respectively, with immune response evaluation as an additional objective. We ex vivo expanded highly pure NKT cells (mean \pm s.d., $94.7 \pm 3.8 \%$ ) and treated patients with $3 \times 10^{6}$ CAR-NKT cells per square meter of body surface area after lymphodepleting conditioning with cyclophosphamide/fludarabine (Cy/Flu). Cy/Flu conditioning was the probable cause for grade 3-4 hematologic adverse events, as they occurred before CAR-NKT cell infusion, and no dose-limiting toxicities were observed. CAR-NKT cells expanded in vivo, localized to tumors and, in one patient, induced an objective response with regression of bone metastatic lesions. These initial results suggest that CAR-NKT cells can be expanded to clinical scale and safely applied to treat patients with cancer.
\end{abstract}

V $\alpha 24$-invariant NKT cells are a sublineage of innate-like T cells that express the V $\alpha 24-\mathrm{J} \alpha 18$ invariant $\mathrm{T}$ cell receptor $\alpha$-chain and react to glycolipids presented by $C D 1 d^{1}$. In contrast to conventional $\mathrm{T}$ cells that require priming to become functionally competent, NKT cells undergo a unique differentiation program in the thymus through which they acquire NK-like properties, including potent cytotoxicity, rapid cytokine secretion and effective trafficking to tissues ${ }^{2}$. Unlike NK cells, however, NKT cell target recognition is CD1d restricted, analogous to HLA restriction in T cells ${ }^{3}$. Although most tumors are CD1d negative and cannot be directly targeted by NKT cells ${ }^{3}$, NKT cells migrate to tumor sites in response to tumor-derived chemokines ${ }^{4,5}$, and their presence in primary tumors correlates with favorable outcomes in some cancer types ${ }^{4,6}$. Within tumors, NKT cells can kill tumor-associated macrophages ${ }^{5,7}$, which promote tumor growth and metastasis ${ }^{8}$. Moreover, NKT cell activation indirectly promotes NK- and T cell-mediated anti-tumor responses ${ }^{1}$. Indeed, transactivation of CD8 T and NK cells has been a consistent finding in clinical studies designed to activate endogenous NKT cells ${ }^{9}$ or adoptively transfer ex vivo-activated NKT cells into patients with cancer ${ }^{10,11}$. However, the earlier trials summarized in Motohashi et al. ${ }^{10}$ had low frequencies of NKT cells in the infusion products, and the later trial ${ }^{11}$ did not examine NKT cell trafficking to tumor sites.

The innate and adaptive immune-modulating properties of NKT cells are largely absent in the polyclonal $\mathrm{T}$ cell products currently used for cell therapy, and NKT cells might, therefore, be an alternative cellular platform for CAR-redirected immunotherapy $^{12,13}$. However, the low frequency of NKT cells in human peripheral blood mononuclear cells (PBMCs) $(\sim 0.1 \%)$ and even lower prevalence in patients with cancer ${ }^{14}$ have necessitated the development of new methods distinct from those used for T or NK cells to accomplish clinical-scale NKT cell expansion ${ }^{15}$.

Neuroblastoma (NB) cells selectively express high levels of GD2-ganglioside ${ }^{16}$. In a recent clinical trial of $\mathrm{T}$ cells expressing a third-generation GD2-specific CAR, the therapy was well tolerated, but none of the 11 patients with NB achieved an objective response ${ }^{17}$. To develop GD2-CAR-NKT cell therapy, we evaluated a series of constructs encoding a GD2-CAR with or without interleukin (IL)15 , a cytokine that supports NKT cell survival ${ }^{12,18}$. Compared to GD2-CAR-NKT cells, NKT cells expressing a GD2-CAR-IL-15 construct demonstrated superior in vivo persistence, tumor infiltration and anti-tumor activity in metastatic NB mouse models without causing evident toxicity ${ }^{19}$. We, therefore, initiated a first-in-human phase 1 clinical trial evaluating autologous CAR-NKT cells in eligible children with relapsed or refractory NB (eligibility criteria presented in Supplementary Table 1). Primary and secondary objectives were to assess safety and anti-tumor responses, respectively, with immune correlative studies as an additional objective. Here we present an interim analysis of results obtained from the first three patients on this study.

The treatment protocol included lymphodepleting conditioning on days $-4,-3$ and -2 (cyclophosphamide, $500 \mathrm{mg}$ per $\mathrm{m}^{2}$ per dose; fludarabine, $30 \mathrm{mg}$ per $\mathrm{m}^{2}$ per dose) followed by CAR-NKT cell infusion on day 0 (Extended Data Fig. 1). Patients were evaluated at weekly clinic visits. Adverse events (AEs) were collected from the start of lymphodepletion until $28 \mathrm{~d}$ after infusion and described according to the Common Terminology Criteria for Adverse Events, version 4. Patients underwent core biopsies of one involved 
site at 2 weeks after infusion. Response to therapy was assessed at 4 weeks after infusion by standard imaging techniques, including metaiodobenzylguanidine (MIBG), single-photon emission computed tomography (SPECT) and bone marrow aspirates/biopsy (bone marrow-positive patients at time of enrollment).

The first three patients were male, ages 12, 12 and 6 years, with stage $\mathrm{M}$ disease according to the International Neuroblastoma Risk Group, owing to multifocal metastases (Extended Data Fig. 2) ${ }^{20}$. These patients had relapsed or refractory disease despite standard upfront and additional salvage therapies. The patients first under-

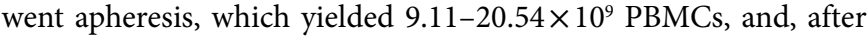
positive selection, $0.4-1.79 \times 10^{6} \mathrm{NKT}$ cells were collected (Extended Data Fig. 3a and Supplementary Table 2). After stimulation with alpha-galactosylceramide-pulsed irradiated PBMCs and expansion, the final products reached $96.5 \%, 97.17 \%$ and $90.36 \%$ (mean, $94.7 \%$ ) NKT cell purity for the three patients, respectively (Extended Data Fig. $3 \mathrm{~b}$ and Supplementary Table 2). In contrast, in the most successful clinical-scale NKT cell expansion to date in which isolated NKT cells were, instead, stimulated with CD3/CD28 agonistic antibodies, the mean NKT purity was $54.4 \%$ across nine patients (range, $13-87 \%)^{11}$. CAR-positive cells accounted for $34.71 \%, 20.18 \%$ and $70.4 \%$ of the NKT cell population for the three patients, respectively (Extended Data Fig. 3b and Supplementary Table 2). The infused products in all patients were predominantly $\mathrm{CD} 4$ positive and contained large fractions of cells positive for CD62L (40.0-78.5\%), a marker linked to in vivo persistence of CAR-NKT cells in preclinical models ${ }^{15}$ (Supplementary Fig. 1).

The first three patients represent all patients on dose level 1: $3 \times 10^{6}$ GD2-CAR-IL- 15 NKT cells per $\mathrm{m}^{2}$. The CAR-NKT cells were well tolerated, and no dose-limiting toxicities (DLTs), including cytokine release syndrome (CRS) or neurotoxicities, were observed. $\mathrm{Cy} /$ Flu was the probable cause of grade 3-4 AEs, including reductions in total white blood cells and red blood cells, as well as absolute lymphocyte, monocyte and platelet counts, as they were present by day 0 before CAR-NKT cell infusion (Extended Data Figs. 4 and 5a). This is consistent with AEs observed in patients with NB after lymphodepletion who were treated with GD2-CAR T cells ${ }^{17}$. Analysis of major lymphoid and myeloid subsets (additional objectives) revealed several notable differences between patients. Patient 1 showed limited recovery of any subsets except NK cells; patient 2 showed expansion of NK cells, Tregs and monocytes after week 2; and patient 3 retained residual CD8 T cells after lymphodepletion that increased by week 4 (Extended Data Fig. 5b).

In all patients, we detected an increase above baseline in peripheral NKT cell frequency (Fig. 1a and Extended Data Fig. 6) and absolute number (Fig. 1b), both of which were sustained until the end of the 4-week evaluation period. CAR-NKT cells were also detected in all patients, and similar kinetics were found by flow cytometry and quantitative polymerase chain reaction (qPCR) (Fig. 1c,d). Absolute CAR-NKT cell numbers were lowest in patient 1 and highest in patient 3, in whom CAR-NKT cells expanded throughout the 4-week evaluation. Products for patients 1, 2 and 3 underwent 15, 14 and 9 days ex vivo culturing, respectively, which might have influenced the in vivo expansion of the infused cells ${ }^{21}$. Single-cell RNA sequencing (scRNA-seq) analysis of the infused
CAR-NKT cell products revealed the presence of nine distinct gene expression clusters that were present in all three patients, albeit at different frequencies (Fig. 1e, Supplementary Fig. 2 and Supplementary Table 3). CAR transgene expression was concentrated in cluster 3, which was also enriched in gene sets associated with central memory versus effector memory and activation versus exhaustion (Fig. 1f-h). We observed that the frequency of cluster 3 (CAR-positive and CAR-negative cells) was highest in the product of patient 3 (Supplementary Fig. 3). Cluster 3 was further characterized by co-expression of genes associated with co-stimulation (TNFRSF4, encoding OX40), central memory (SELL, encoding CD62L) and the Wnt pathway (Extended Data Fig. 7a). Flow cytometry confirmed the existence of $\mathrm{CD}_{2} 2 \mathrm{~L}^{+} \mathrm{OX} 40^{+}$and $\mathrm{CD} 62 \mathrm{~L}^{+} \mathrm{LEF}^{+}$ (a Wnt pathway transcription factor) NKT cell subsets in all products (Extended Data Fig. 7b,c). We also observed high expression levels of metabolic genes in cluster 3 (Extended Data Fig. 8a). A bioenergetics analysis demonstrated that cells from patients 2 and 3 had a significantly higher rate of glycolysis $(P<0.01)$ and glycolytic capacity $(P<0.001)$ than cells from patient 1 , and that cells from patient 2 had a higher spare respiratory capacity than cells from patient $3(P<0.01)$ (Extended Data Fig. 8b-e).

Next, we performed a serial tumor challenge assay in which NB cells were replenished every $5 \mathrm{~d}$ for five rounds, as previously described ${ }^{19}$ (Extended Data Fig. 9a). After the third round, CAR-NKT cells from patient 1 progressively lost cytotoxic activity, whereas those from patients 2 and 3 retained the ability to kill NB cells through the fifth round $(P<0.01$; Extended Data Fig. 9b,c). CAR-NKT cells from patients 2 and 3 also showed enhanced proliferative response compared to cells from patient 1 throughout the first four rounds of co-culture ( $P<0.05$; Extended Data Fig. 9d).

Homeostatic cytokines, including IL-7 and IL-15, promote the expansion of adoptively transferred lymphocytes after lymphodepletion $^{17,22}$. In these patients, peripheral blood IL-15 levels increased around the time of CAR-NKT cell infusion and returned to approximately baseline by week 4, whereas IL-7 remained close to baseline throughout (Extended Data Fig. 10). The observed pattern of serum IL-15 changes is consistent with the expected effect of the lymphodepleting conditioning and is unlikely to be related to the infused GD2-CAR-IL-15 NKT cells. This is also consistent with preclinical data in which serum IL-15 remained undetectable in NSG mice treated with a dose of GD2-CAR-IL-15 NKT cells 1,000-fold higher than that received by patients on this study ${ }^{19}$.

To evaluate CAR-NKT cell tumor localization, we performed flow cytometry analyses on a post-infusion biopsy specimen from patient 1 . Within the small population of tumor-infiltrating lymphocytes, NKT cells were present at a higher frequency than detected in peripheral blood lymphocytes; notably, $85.7 \%$ of tumor-infiltrating NKT cells expressed the GD2-CAR (Fig. 2a). The CAR transgene was undetectable by qPCR in a small quantity of DNA isolated from this sample. Although not measurable by flow cytometry, qPCR for the CAR transgene detected 60.4 and 23.7 copies per microgram of DNA in patients 2 and 3, respectively (Supplementary Table 4 ). We also examined the ability of CAR-NKT cells to penetrate the bone marrow in patient 1 , the only patient with bone marrow metastases. As with the solid tumor

Fig. 1 | CAR-NKT cells expand and persist in patients with relapsed or refractory NB. a, Flow cytometry plots showing pre- and post-infusion NKT cell frequency and GD2-CAR expression in peripheral blood samples from patient 3. Histogram shows CAR expression in week 3 after infusion of NKT cells (red) compared to T cells (gray) as a negative control. SSC, side scatter. b. Absolute NKT cell numbers in peripheral blood as determined by flow cytometry and absolute lymphocyte counts for the three patients. c, Absolute number of circulating CAR-NKT cells as determined by flow cytometry. d, Fold change in GD2-CAR transgene DNA copy number per milliliter of peripheral blood compared to 3-hour post-infusion baseline as determined by qPCR. e, Unsupervised clustering of single-cell gene expression visualized using the UMAP algorithm in pre-infusion products. Each point represents a single cell (9,323 cells total); cells are colored by cluster. f, Expression of GD2-CAR transgene after secondary analysis (using primers specific to the GD2-CAR) overlaid on the UMAP two-dimensional projection. $\mathbf{g}$, Comparative gene expression of central memory versus effector memory and (h) activation versus exhaustion markers from reference gene signatures (Methods) overlaid on the UMAP two-dimensional projection. 
biopsy, we found a higher percentage of NKT cells in the bone marrow metastases than in the peripheral blood, but, in contrast, only a small fraction of these cells expressed the GD2-CAR (33.4 copies per microgram of DNA by qPCR) (Fig. 2b and Supplementary
Table 4). We observed higher levels of GD2 expression in NB cells in the tumor biopsy of patient 3 and in the bone marrow of patient 1 than in the tumor biopsy of patient 1 , suggesting the possibility of antigen escape (Supplementary Fig. 4).

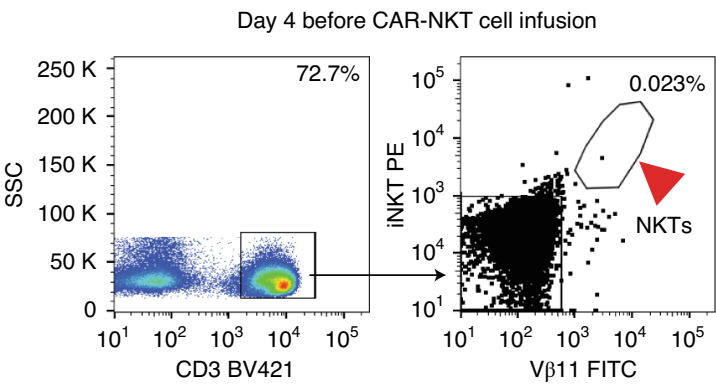

Week 3 after CAR-NKT cell infusion
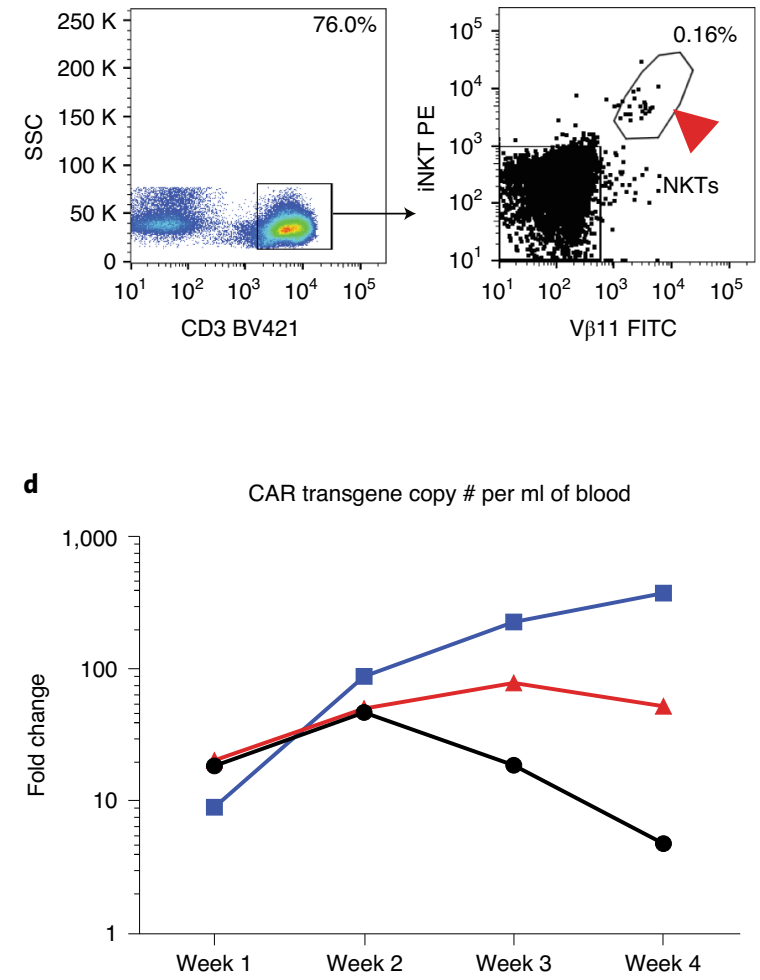

b
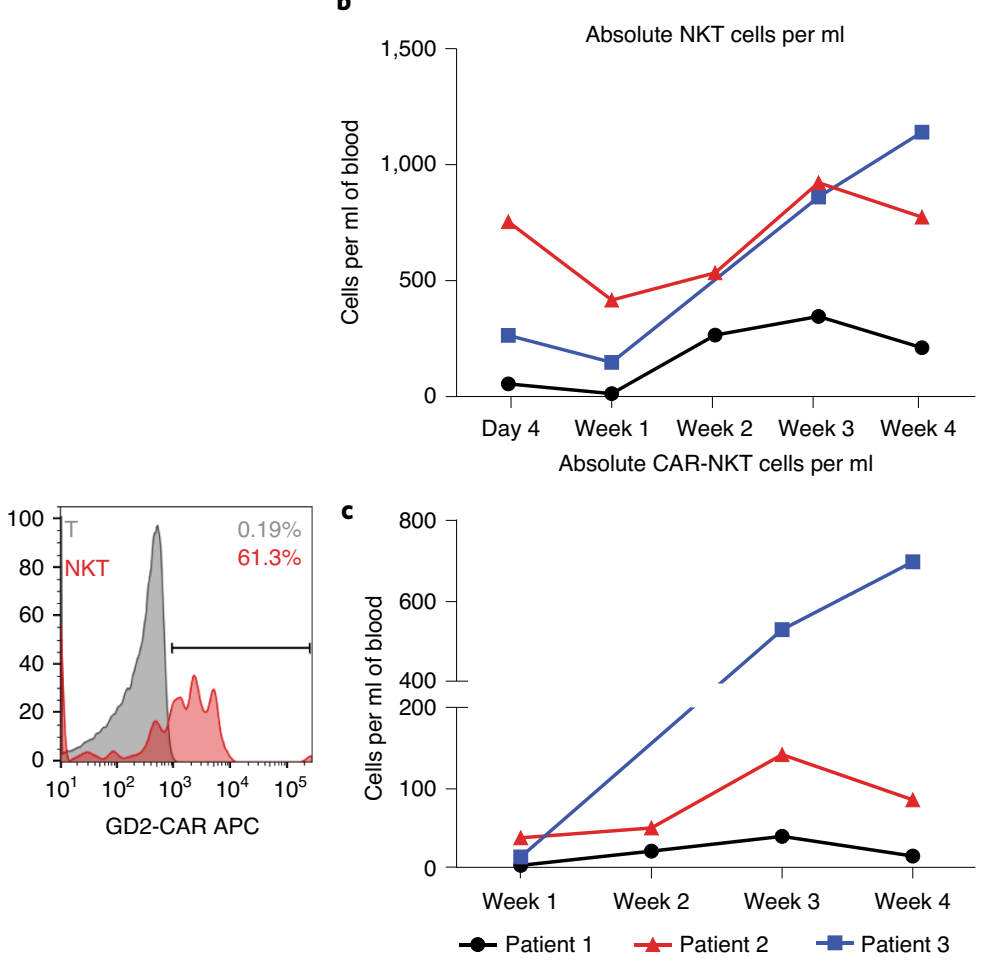

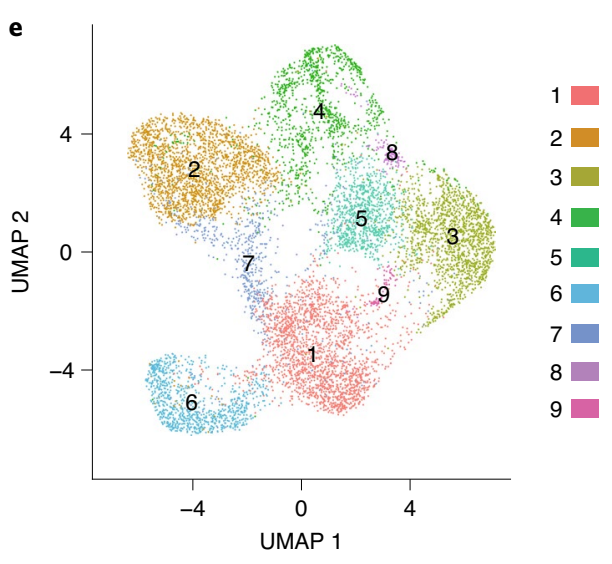

$\mathbf{f}$

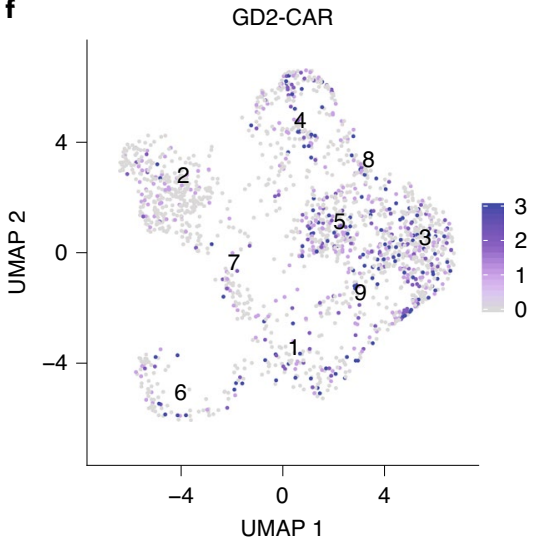

g

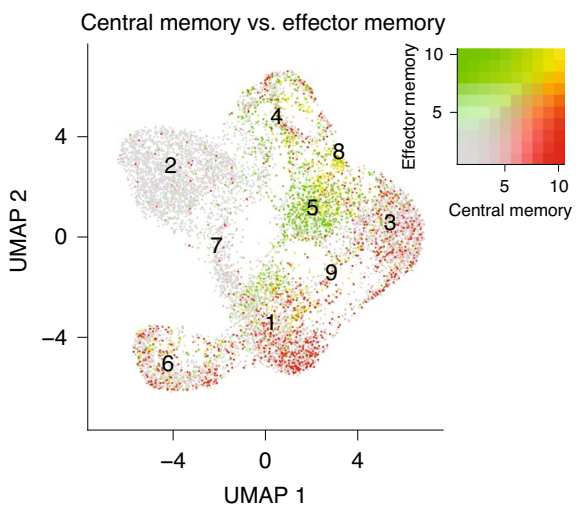

h

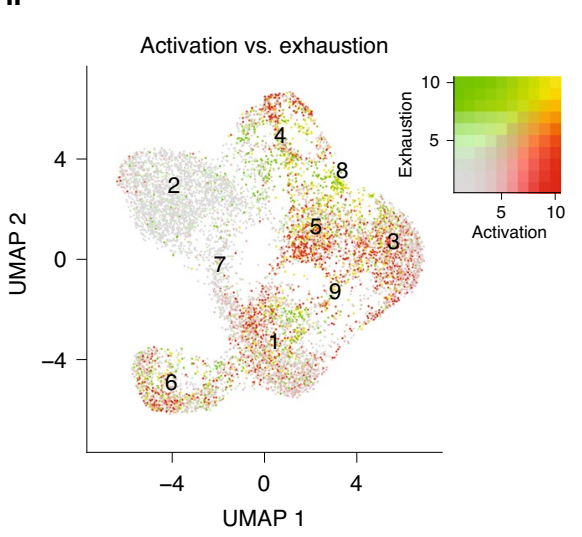




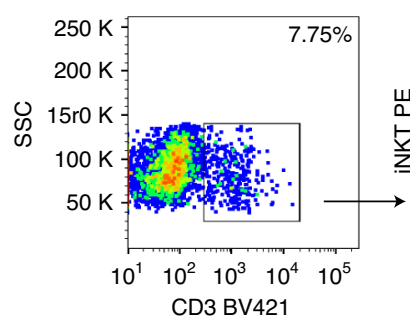

b

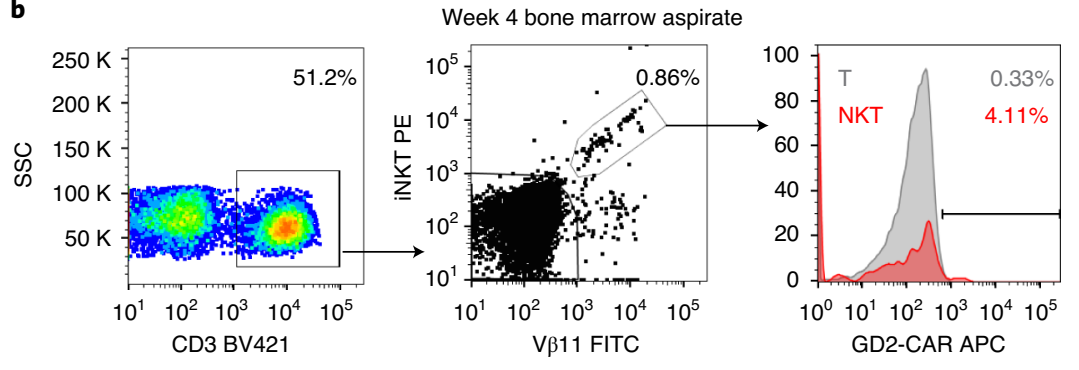

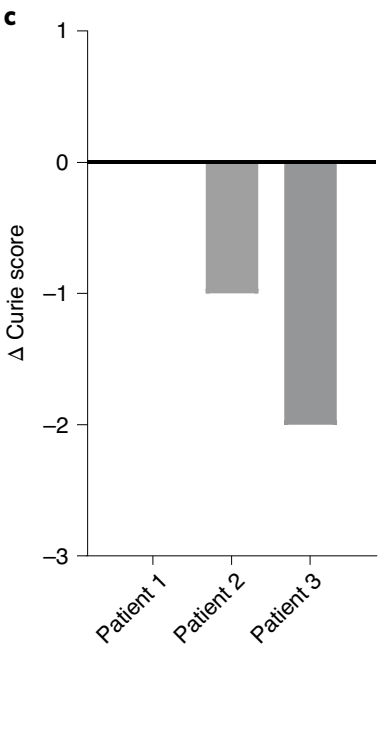

d

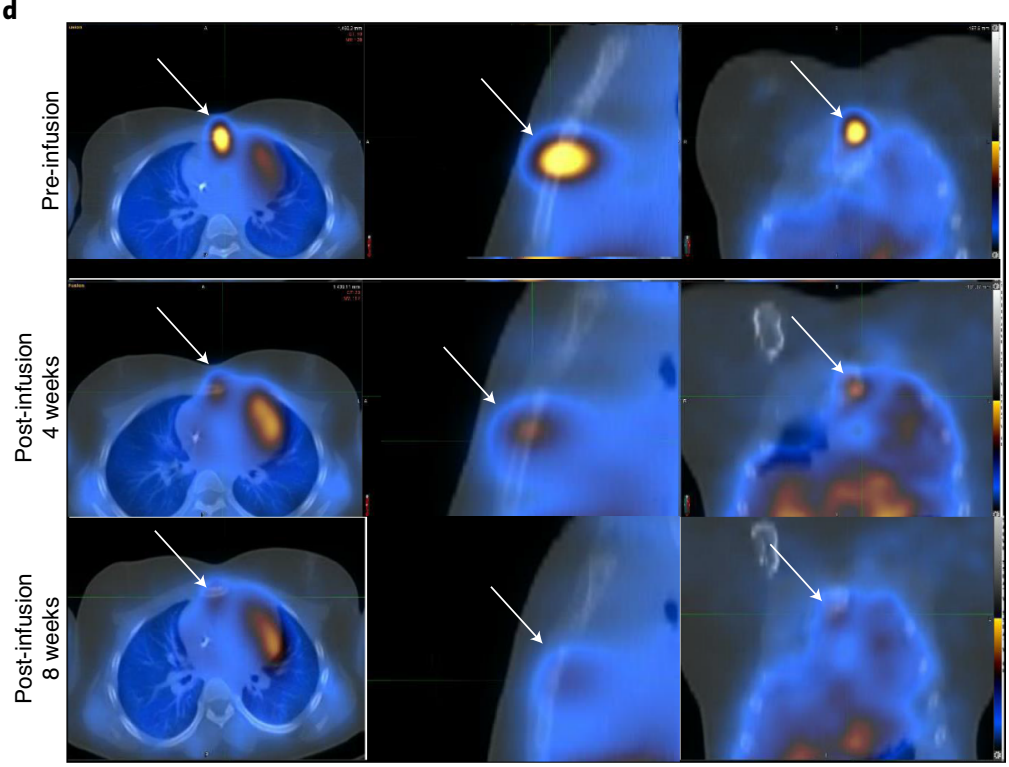

Fig. 2 | CAR-NKT cells infiltrate tumor sites and mediate tumor regression. a, Flow cytometry plots showing NKT cells as a percentage of total tumor-infiltrating lymphocytes 2 weeks after infusion in a biopsy from patient 1. Histogram shows CAR expression in these NKT cells (red) compared to T cells (gray) as a negative control. b. Flow cytometry plots showing NKT cells in bone marrow aspirate from patient 14 weeks after infusion. Histogram shows CAR expression in these NKT cells (red) compared to T cells (gray) as a negative control. c, Change in Curie score at 4 weeks after infusion versus before infusion for the three patients. d, Merged SPECT and MIBG uptake scans showing a metastatic sternum lesion from patient 2 at indicated time points.

Therapeutic responses, the secondary objective of this study, were examined using the International Neuroblastoma Response Criteria (INRC) ${ }^{23}$ and by comparing pre- and post-infusion NB burden using Curie scoring, which has prognostic value ${ }^{24}$. Patient 1 had stable disease as determined by the INRC, and his Curie score did not change after CAR-NKT cell treatment (Fig. 2c). Patient 3 had stable disease, and his Curie score decreased from 7 to 5. Patient 2 had a partial response and a change in Curie score from 2 to 1 ; SPECT- and MIBG-merged scan images revealed a dramatic reduction in the size and MIBG uptake of a bone metastasis in that patient (Fig. 2d). The patient consequently received salvage therapy and achieved a complete response that lasted approximately 6 months (Extended Data Fig. 2).

In conclusion, our initial results demonstrate that NKT cells can be expanded to clinical scale with high purity, genetically engineered to express a CAR and used to safely treat patients with NB.
In addition, we found that CAR-NKT cells can be detected in the peripheral blood where they expand after infusion, traffic to bone metastases and the bone marrow and induce tumor regression in heavily pretreated patients with relapsed or refractory disease. This interim analysis suggests that the in vivo persistence and anti-tumor potential of CAR-NKT cells could be influenced by cell characteristics, including retention of the central memory program, metabolic fitness and resistance to exhaustion, as well as other factors, including the duration of product manufacturing and retention of target antigen on the tumor cells. These hypotheses are being tested while the study continues to enroll patients.

\section{Online content}

Any methods, additional references, Nature Research reporting summaries, source data, extended data, supplementary information, acknowledgements, peer review information; details of 
author contributions and competing interests; and statements of data and code availability are available at https://doi.org/10.1038/ s41591-020-1074-2.

Received: 10 March 2020; Accepted: 21 August 2020; Published online: 12 October 2020

\section{References}

1. Godfrey, D. I., MacDonald, H. R., Kronenberg, M., Smyth, M. J. \& Van Kaer, L. NKT cells: what's in a name? Nat. Rev. Immunol. 4, 231-237 (2004).

2. Savage, A. K. et al. The transcription factor PLZF directs the effector program of the NKT cell lineage. Immunity 29, 391-403 (2008).

3. Metelitsa, L. S. Anti-tumor potential of type-I NKT cells against CD1d-positive and CD1d-negative tumors in humans. Clin. Immunol. 140, 119-129 (2011).

4. Metelitsa, L. S. et al. Natural killer T cells infiltrate neuroblastomas expressing the chemokine CCL2. J. Exp. Med. 199, 1213-1221 (2004).

5. Song, L. et al. Valpha24-invariant NKT cells mediate antitumor activity via killing of tumor-associated macrophages. J. Clin. Invest. 119, 1524-1536 (2009).

6. Tachibana, T. et al. Increased intratumor Valpha24-positive natural killer T cells: a prognostic factor for primary colorectal carcinomas. Clin. Cancer Res. 11, 7322-7327 (2005).

7. Cortesi, F. et al. Bimodal CD40/Fas-dependent crosstalk between iNKT cells and tumor-associated macrophages impairs prostate cancer progression. Cell Rep. 22, 3006-3020 (2018).

8. Noy, R. \& Pollard, J. W. Tumor-associated macrophages: from mechanisms to therapy. Immunity 41, 49-61 (2014).

9. Chang, D. H. et al. Sustained expansion of NKT cells and antigen-specific T cells after injection of alpha-galactosyl-ceramide loaded mature dendritic cells in cancer patients. J. Exp. Med. 201, 1503-1517 (2005).

10. Motohashi, S., Okamoto, Y., Yoshino, I. \& Nakayama, T. Anti-tumor immune responses induced by iNKT cell-based immunotherapy for lung cancer and head and neck cancer. Clin. Immunol. 140, 167-176 (2011).

11. Exley, M. A. et al. Adoptive transfer of invariant NKT cells as immunotherapy for advanced melanoma: a phase I clinical trial. Clin. Cancer Res. 23, 3510-3519 (2017)

12. Heczey, A. et al. Invariant NKT cells with chimeric antigen receptor provide a novel platform for safe and effective cancer immunotherapy. Blood 124, 2824-2833 (2014).
13. Rotolo, A. et al. Enhanced anti-lymphoma activity of CAR19-iNKT cells underpinned by dual CD19 and CD1d targeting. Cancer Cell 34, 596-610 (2018)

14. Tahir, S. M. et al. Loss of IFN-gamma production by invariant NK T cells in advanced cancer. J. Immunol. 167, 4046-4050 (2001).

15. Tian, G. et al. CD62 $\mathrm{L}^{+}$NKT cells have prolonged persistence and antitumor activity in vivo. J. Clin. Invest. 126, 2341-2355 (2016).

16. Schulz, G. et al. Detection of ganglioside GD2 in tumor tissues and sera of neuroblastoma patients. Cancer Res. 44, 5914-5920 (1984).

17. Heczey, A. et al. CAR T cells administered in combination with lymphodepletion and PD-1 inhibition to patients with neuroblastoma. Mol. Ther. 25, 2214-2224 (2017)

18. Liu, D. et al. IL-15 protects NKT cells from inhibition by tumor-associated macrophages and enhances antimetastatic activity. J. Clin. Invest. 122, 2221-2233 (2012).

19. Xu, X., et al. NKT cells co-expressing a GD2-specific chimeric antigen receptor and IL-15 show enhanced in vivo persistence and antitumor activity against neuroblastoma. Clin. Cancer Res. 25 7126-7138 (2019).

20. Cohn, S. L. et al. The international neuroblastoma risk group (INRG) classification system: an INRG task force report. J. Clin. Oncol. 27, 289-297 (2009).

21. Ghassemi, S. et al. Reducing ex vivo culture improves the antileukemic activity of chimeric antigen receptor (CAR) T cells. Cancer Immunol. Res. 6, 1100-1109 (2018).

22. Gattinoni, L. et al. Removal of homeostatic cytokine sinks by lymphodepletion enhances the efficacy of adoptively transferred tumor-specific CD8+ T cells. J. Exp. Med. 202, 907-912 (2005).

23. Park, J. R. et al. Revisions to the international neuroblastoma response criteria: a consensus statement from the National Cancer Institute Clinical Trials Planning Meeting. J. Clin. Oncol. 35, 2580-2587 (2017).

24. Decarolis, B. et al. Iodine-123 metaiodobenzylguanidine scintigraphy scoring allows prediction of outcome in patients with stage 4 neuroblastoma: results of the Cologne Interscore Comparison Study. J. Clin. Oncol. 31, 944-951 (2013).

Publisher's note Springer Nature remains neutral with regard to jurisdictional claims in published maps and institutional affiliations. 


\section{Methods}

Detailed information on experimental design and reagents can be found in the Life Sciences Reporting Summary.

Study development and design. The 'GD2 Specific CAR and Interleukin-15 Expressing Autologous NKT Cells to Treat Children With Neuroblastoma' (GINAKIT2) phase 1 clinical trial was reviewed and approved by the Protocol Review Committee, the Institutional Biosafety Committee and the Institutional Review Board at Baylor College of Medicine, as well as the Recombinant DNA Advisory Board of the National Institutes of Health and the US Food and Drug Administration (FDA). The study was conducted at Texas Children's Hospital, Baylor College of Medicine, in Houston, Texas, in accordance with Declaration of Helsinki principles. All participants and/or their legal guardians provided written informed consent/assent upon enrollment and before administration of the cells. The GINAKIT2 study is a phase 1 clinical trial using standard $3+3$ dose escalation, as described in the clinical trial protocol (Supplementary Information). Patients were enrolled between August 20, 2018, and July 29, 2019. Eligibility inclusion criteria for treatment included relapsed or refractory high-risk NB, age older than 1 year and less than 21 years, appropriate bone marrow, liver, renal, cardiac and respiratory function and absence of human anti-mouse antibodies (in patients with previous exposure to murine antibodies). Exclusion criteria included rapidly progressing disease, receiving other investigational drugs, immunosuppressive therapy or history of severe previous toxicity to cyclophosphamide or fludarabine. Full eligibility criteria are listed in Supplementary Table 1.

Clinical safety and outcome assessment. AEs were collected from the start of lymphodepletion until $28 \mathrm{~d}$ after infusion and described according to the Common Terminology Criteria for Adverse Events, version 4. A DLT was defined as: 1) any hematological or non-hematological grade 3-5 toxicity that is considered to be primarily related to the CAR-NKT cells; 2) CRS and neurological toxicities grade 3 and 4; expected reactions seen with the use of CAR-based immunotherapy, such as fever or hypotension lasting less than $72 \mathrm{~h}$, will not be considered DLTs; symptoms lasting more than $72 \mathrm{~h}$ will be considered DLTs; 3 ) any other unexpected grade 3 or higher toxicity thought to be related to, or resulting from, CRS or due to neurological toxicity is included in the definition of DLT; and 4) grade 3 and 4 CRS or neurological toxicities that are persistent beyond $72 \mathrm{~h}$ will be reported to the FDA in an expedited fashion and will be considered DLTs. Anti-tumor responses were defined by comparing standard three-dimensional imaging (computed tomography and magnetic resonance imaging), MIBG scan and bone marrow aspirates/biopsies from pre- and post-CAR-NKT cell infusion. Anti-tumor responses were examined using the International Neuroblastoma Response Criteria ${ }^{22}$ and by measuring the difference in pre- and post-infusion Curie scoring ${ }^{23}$.

Clinical-grade vector production. The clinical-grade vector encoding the GD2-CAR-IL-15 construct was produced at the current good manufacturing practices (cGMP) facility of the Center for Cell and Gene Therapy at Baylor College of Medicine. Briefly, the SFG plasmid encoding the GD2-CAR-IL-15 construct was transfected into HEK-293T cells, which produce Gibbon ape leukemia virus pseudotype and Moloney murine leukemia virus-derived gamma-retroviral particles. The clinical vector was validated, tested according to cGMP guidance, cryopreserved and stored at $-80^{\circ} \mathrm{C}$ until use.

CAR-NKT cell manufacturing. PBMCs were isolated from peripheral blood leukapheresis products using Ficoll gradient purification, and NKTs were isolated using a special lot of anti-iNKT microbeads (Miltenyi Biotec) (tested according to FDA guidelines by BioReliance) and the CliniMACS instrument (Miltenyi Biotec). Enriched NKT cells were then stimulated with cGMP-grade alpha-galactocylceramide and (GlycoSyn)-pulsed PBMCs and cultured with IL-2 and IL-21 (NCI, $200 \mathrm{U} \mathrm{ml}^{-1}$ and CellGenix, $10 \mathrm{ng} \mathrm{ml}^{-1}$, respectively). Culture supplementation with IL-21 is used to increase the frequency and functionality of $\mathrm{CD}^{2} \mathrm{~L}^{+} \mathrm{NKT}$ cells ${ }^{25}$. Cells were transduced with retroviral particles encoding the GD2-CAR-IL-15 construct in RetroNectin-coated plates (Takara Bio) for $72 \mathrm{~h}$ and then washed, replated and cultured at $38^{\circ} \mathrm{C}$ for 9-15 $\mathrm{d}$. CAR-NKT cell products (patients 1 and 2) that did not have sufficient numbers on day 10 were restimulated with alpha-galactocylceramide-pulsed PBMCs and expanded until sufficient numbers were met. At the end of manufacture, the product was evaluated using trypan blue staining and flow cytometry. Additionally, GD2-CAR transgene copy number was determined by qPCR.

Transgene copy number assessment. GD2-CAR transgene copy number was evaluated in peripheral blood and tumor biopsy samples. Genomic DNA was extracted using the QIAamp DNA Blood Minikit (Qiagen) according to the manufacturer's instructions, and transgene copy number was measured by TaqMan qPCR gene expression assay using primers (forward: 5'-GCTGCACCA ACTGTATCCATCTT-3'; reverse: 5'-GGTCCAGACTGCTGAAGCT- $3^{\prime}$ ) and probe (5'-CACCCGACCCACCACC-3') sequences complementary to the GD2-CAR sequence (Applied Biosystems). qPCR was performed in $25-\mu$ l reaction volumes using the ABI 7900HT Sequence Detection System (Applied
Biosystems). Copy number was normalized to $1 \mu \mathrm{g}$ of DNA isolated from PBMCs. Lymphodepleting chemotherapy with Cy/Flu decreases total white blood cell numbers, resulting in an artificial increase in transgene copy number as measured by PCR when normalized to DNA only; therefore, transgene copy number was calculated per milliliter of peripheral blood.

Multiplex cytokine assay. Serum cytokine levels were measured using the Milliplex MAP magnetic bead-based human cytokine pre-mixed 38-plex kit (EMD Millipore) on the Luminex 200 system with xPONENT software (Luminex) according to the manufacturer's instructions.

Flow cytometry analyses. GD2-CAR NKT cells were detected in peripheral blood and patient biopsy samples using the following antibodies: FITC-conjugated anti-TCR V $\beta 11$ (clone C21, Beckman Coulter), PE-conjugated anti-iNKT (6B11, BD Biosciences), BV421-conjugated anti-CD3 (SK7, BD Biosciences) and an anti-idiotype GD2-CAR antibody (1A7), produced in-house using murine hybridoma HB-11786 (ATCC) and custom conjugated with APC (BioLegend). Evaluation of patient products also included PE-Cy7-conjugated anti-CD62L (DREG56) and APC-H7-conjugated anti-CD4 (SK3), both from $\mathrm{BD}$ Biosciences. To evaluate lymphoid subsets, the following antibodies were used: FITC-conjugated anti-CD3 (SK7), APC-H7- or APC-conjugated anti-CD8 (SK1), BV510- or PE-conjugated anti-CD4 (SK3), PE-Cy7-conjugated anti-CD25 (2A3), BV421-conjugated anti-CD127 (HIL-7R-M21), PE-Cy7-conjugated anti-CD56 (NCAM16.2) and BV421-conjugated anti-CD19 (SJ25C1), all from $\mathrm{BD}$ Biosciences. To evaluate myeloid subsets, the following antibodies were used: PE-Cy7-conjugated anti-CD11b (ICRF44), BV510-conjugated anti-HLA-DR (G46-6), APC-H7-conjugated anti-CD14 (MФP9), PE-conjugated anti-CD16 (B73.1) and FITC-conjugated anti-CD15 (MMA), all from BD Biosciences. Intracellular staining was performed using the Foxp3/transcription factor staining buffer set (eBioscience). Dead cells were excluded using fixable viability dye eFluor 780 (eBioscience), and the following monoclonal antibodies were used: FITC-conjugated anti-CD3 (UCHT1), BV605-conjugated anti-CD4 (RPA-T4), PE-Cy7-conjugated anti-CD62L (DREG-56) and BV421-conjugated anti-CD134 (ACT35), all from BD Biosciences, as well as AF647-conjugated anti-GD2 CAR 1A7 and PE-conjugated anti-LEF1 C12A5 (Cell Signaling Technology). To evaluate NB cells, the following antibodies were used: PE-Cy7-conjugated anti-CD56 (NCAM16.2), FITC-conjugated GD2 (14.G2A) and PerCP-conjugated CD45 (2D1), all from BD Biosciences. Sequential flow cytometry gating strategies can be found in Supplementary Fig. 5. Samples were analyzed on an LSR-II five-laser flow cytometer using BD FACSDiva software, version 6.0, and FlowJo 10.6.1 (BD Biosciences).

Metabolic analyses. Oxygen consumption rates and extracellular acidification rates were measured in pre-adjusted pH 7.4 Seahorse XF RPMI medium, supplemented with $2 \mathrm{mM}$ L-glutamine alone (glycolysis stress test) or associated with $10 \mathrm{mM}$ glucose and $1 \mathrm{mM}$ pyruvate (mito stress test), using a 96-well XFe Extracellular Flux Analyzer (all from Agilent Technologies). Measurements were performed with 200,000 cells per well under basal conditions and in response to $1.5 \mu \mathrm{M}$ oligomycin, $2 \mu \mathrm{M}$ fluoro-carbonyl cyanide phenylhydrazone, $0.5 \mu \mathrm{M}$ rotenone, $0.5 \mu \mathrm{M}$ antimycin A or $50 \mathrm{mM}$ 2-deoxy-D-glucose (all from Sigma-Aldrich).

Serial tumor challenge assay. Patient CAR-NKT cells and CHLA-255 NB cells transduced with green fluorescent protein were co-cultured in a 24 -well plate using fresh culture medium at a 1:1 effector-to-target (E:T) ratio, respectively. Every other day, $50 \mathrm{U} \mathrm{ml}^{-1}$ of IL-2 was added. Five days later, cells were harvested, quantified by trypan blue exclusion and analyzed for NKT and NB cell markers by flow cytometry. CAR-NKT cells were then replated at a 1:1 E:T ratio with fresh NB cells in fresh cell culture medium to start the next round of tumor co-culture. The process was repeated for five rounds.

Statistics for in vitro functional experiments. Two-way analysis of variance with Tukey's correction for multiple comparisons was used to determine the effect of two independent variables on a continuous dependent variable (Extended Data Figs. 8 and 9). An unpaired $t$-test was used to compare the averages of two independent groups (Extended Data Fig. 10e; patient 2 versus patient 3 with three technical replicates each). Statistics were computed using GraphPad Prism 7.0 (GraphPad Software). Differences were considered significant when the $P$ value was less than 0.05 .

Cell hashing and scRNA-seq library construction. Pre-infusion products from patients 1-3 were labeled with different barcoded TotalSeq-C Hashtag antibodies (anti- $\beta 2 \mathrm{M}$ (clone $2 \mathrm{M} 2$ ) and CD298 (clone LNH-94), BioLegend) for $30 \mathrm{~min}$ at room temperature in RPMI (Life Technologies) + 2\% FBS (Life Technologies). Cells were then washed three times in PBS (Life Technologies) + BSA 0.04\% (VWR). Finally, cells were pooled and loaded into the Chromium Next GEM Single Cell 5' Library and Gel Bead Kit version 1.1 workflow (10× Genomics). Single-cell RNA and cell hashing ${ }^{26}$ library preparation was performed according to the manufacturer's instructions. Libraries were sequenced by GENEWIZ on the NovaSeq 6000 System using the S4 $2 \times 150$ kit (Illumina). 
scRNA-seq data processing. Two separate experiments were performed. The samples were processed similarly, and data were integrated as follows. Briefly, matrices were produced using the Cell Ranger Single-Cell Software Suite (v3.1.0) with a human transcriptome reference (GRCh38) with the following modifications: 1) the GD2-CAR construct sequence was added;2) to avoid an erroneous cross-mapping of GD2-CAR scFv transcripts to immunoglubulin genes, the immunoglubulin sequences were removed from the reference. Data analysis was performed using Seurat (v3.1.1), which is publicly available. Product samples were de-hashed, and singlets were extracted by applying HTODemux ${ }^{27}$ on CLR-normalized data with 0.95 as the positive quantile. RNA data were normalized using SCTransform ${ }^{28}$ regressed on percent of mitochondrial genes and integrated using the FindIntegrationAnchors function and the CCA method ${ }^{29}$. Dimensionality was reduced using the Uniform Manifold Approximation and Projection (UMAP) algorithm. Differential gene expression was performed using the Seurat function FindAllMarkers default settings; a minimum fraction of cells expressed a gene of $25 \%$ $(\mathrm{mn} . \mathrm{pct}=0.25)$ and $\log _{2}$ fold change of $0.2(\operatorname{logfc}$.threshold $=0.2)$. Comparative gene expression of central memory versus effector memory and activation versus exhaustion markers was performed using published reference gene signatures ${ }^{30-33}$.

GD2-CAR capture from scRNA-seq complementary DNA library. To increase capture rates of the GD2-CAR construct in the single-cell RNA sequencing experiment, we designed a hemi-nested PCR approach that enables the capture of the $10 \times$ cell barcode and the GD2-CAR. Briefly, after complementary DNA PCR amplification, $2 \mu \mathrm{l}$ were used for a first PCR amplification using the primers (forward: $5^{\prime}$-AATGATACGGCGACCACCGAGA TCTACACTCTTTCCCTACACGACGCTC-3' (10× Genomics) and reverse: 5'-CTGGCTTCTGCAGGTACCAATG-3') using Kapa HiFi Polymerase (Kapa Biosystems) and purified using $0.8 \times$ reaction volume SPRI beads (Beckman Coulter). The product of the first PCR was further amplified by a second PCR using the primers (forward: 5' -AATGATACGGCGACCACCGAGATCT-3' (10× Genomics) and reverse: $5^{\prime}$-CTCGTGGGCTCGGAGATGTGTATAAGAG ACAGGGGTCAGCAAAATATCTCTAGAGCACTGG-3') using Kapa HiFi Polymerase and purified using $0.8 \times$ reaction volume SPRI beads. Finally, the GD2-CAR library was indexed using the primers (forward: $5^{\prime}$-AATGATACGGCGACCACCGAGATC TACACTCTTTCCCTACACGACGCTC-3' (10× Genomics) and reverse: 5'-CAAGCAGAAGACGGCATACGAGATTGTGTATAGTCTCGTGGGCTCGG $-3^{\prime}$ ) using Kapa HiFi Polymerase and purified using $0.7 \times$ reaction volume SPRI beads. Libraries were sequenced by GENEWIZ on a NovaSeq 6000 System using the S4 $2 \times 150$ kit (Illumina). Sequencing data were aligned to the GD2-CAR construct using Bowtie2 (ref. ${ }^{33}$ ). Reads that were successfully aligned were then assigned to cells by matching their barcode against the CellRanger's barcode whitelist, without allowing for any base mismatch. All reads belonging to one cell were treated as vertices in a graph and were connected by an edge if the hamming distance between their unique molecular identifier was 0 or 1 . All reads belonging to a connected component of this graph were treated as PCR duplicates and counted as a single GD2-CAR RNA molecule.

Reporting Summary. Further information on research design is available in the Nature Research Reporting Summary linked to this article.

\section{Data availability}

All requests for raw and analyzed data and materials will be promptly reviewed by the Baylor College of Medicine Licensing Group to verify whether the request is subject to any intellectual property or confidentiality obligations. Patient-related data not included in the paper were generated as part of the clinical trial and might be subject to patient confidentiality restrictions. Any data and materials that can be shared will be released via a material transfer agreement. All raw data for single-cell sequencing have been deposited in the Gene Expression Omnibus with the accession code GSE154037. Source data are provided with this paper.

\section{References}

25. Ngai, H. et al. IL-21 selectively protects CD62L $\mathrm{L}^{+}$NKT cells and enhances their effector functions for adoptive immunotherapy. J. Immunol. 201, 2141-2153 (2018).
26. Stoeckius, M. et al. Cell hashing with barcoded antibodies enables multiplexing and doublet detection for single cell genomics. Genome Biol. 19, 224 (2018).

27. Stuart, T. et al. Comprehensive integration of single-cell data. Cell 177, 1888-1902 (2019).

28. Hafemeister, C. \& Satija, R. Normalization and variance stabilization of single-cell RNA-seq data using regularized negative binomial regression. Genome Biol. 20, 296 (2019).

29. Butler, A., Hoffman, P., Smibert, P., Papalexi, E. \& Satija, R. Integrating single-cell transcriptomic data across different conditions, technologies, and species. Nat. Biotechnol. 36, 411-420 (2018).

30. Guo, X. et al. Global characterization of T cells in non-small-cell lung cancer by single-cell sequencing. Nat. Med. 24, 978-985 (2018).

31. Zhang, L. et al. Lineage tracking reveals dynamic relationships of $\mathrm{T}$ cells in colorectal cancer. Nature 564, 268-272 (2018).

32. Yost, K. E. et al. Clonal replacement of tumor-specific T cells following PD-1 blockade. Nat. Med. 25, 1251-1259 (2019).

33. Langmead, B. \& Salzberg, S. L. Fast gapped-read alignment with Bowtie 2. Nat. Methods 9, 357-359 (2012).

\section{Acknowledgements}

The authors are grateful to M. Brenner, H. Heslop and personnel from the cGMP facility at the Center for Cell and Gene Therapy for manufacturing CAR-NKT cells; A. Sher, K. Kukreja and P. Srivaths; staff of the Flow Cytometry Core Laboratory of the Texas Children's Cancer and Hematology Center; P. Saha for excellent technical assistance; and E. Chomsky, E. Kiner and H. Sharim for help with scRNA-seq. This work was supported by grants and contracts from Alex's Lemonade Stand Foundation for Childhood Cancer, Kuur Therapeutics (to L.S.M. and A.H.), the American Cancer Society (to A.H.), Cookies for Kids' Cancer Foundation (to L.S.M.) and the Cancer Prevention and Research Institute of Texas (Baylor College of Medicine Comprehensive Cancer Center Training Program, RP160283, to H.N.).

\section{Author contributions}

A.H. and L.S.M. designed the clinical trial. A.H. wrote the clinical protocol and was the principal investigator of the study. G.D., L.S.M., A.H. and B.S. developed and tested the CAR construct selected for the trial. S.R. wrote CAR-NKT cell manufacturing SOPs. S.R., K.L. and M.L. performed CAR-NKT cell manufacturing. A.N.C., H.N. and N.G. performed flow cytometry, O.D. performed qPCR, A.N.C. performed Luminex assay and B.L. performed Taqman RT-PCR. C.N.C.-F. performed bioenergetics analysis. X.X. performed the serial tumor challenge assay. T.R.-S. and A.M. performed scRNA-seq and analyzed data. The manuscript was written by A.H., A.N.C. and L.S.M. and edited by E.J.D.P. All authors discussed and interpreted the results.

\section{Competing interests}

A.H., A.N.C., G.D. and L.S.M are co-inventors on pending patent applications that relate to the use of NKT cells in cancer immunotherapy and have been licensed by Baylor College of Medicine to Kuur Therapeutics for commercial development. Kuur Therapeutics provided research support for this project (to L.S.M.) via a sponsored research agreement with Baylor College of Medicine. A.M., S.R., K.L., M.L., N.G., O.D., B.L., T.R.-S., C.N.C.-F., X.X., H.N., E.J.D.P. and B.S. declare no competing financial interests.

\section{Additional information}

Extended data is available for this paper at https://doi.org/10.1038/s41591-020-1074-2. Supplementary information is available for this paper at https://doi.org/10.1038/ s41591-020-1074-2.

Correspondence and requests for materials should be addressed to A.H. or L.S.M. Peer review information Saheli Sadanand was the primary editor on this article and managed its editorial process and peer review in collaboration with the rest of the editorial team.

Reprints and permissions information is available at www.nature.com/reprints. 


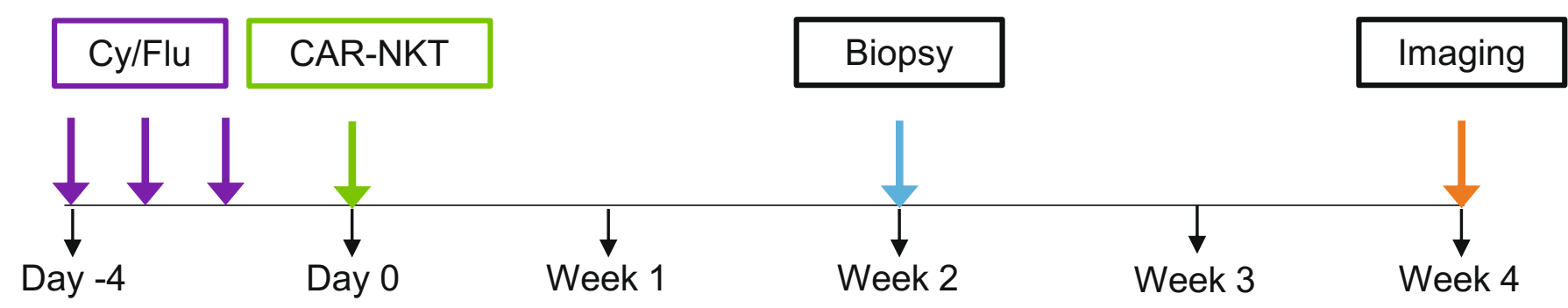

b

\section{CONSORT 2010 Flow Diagram}

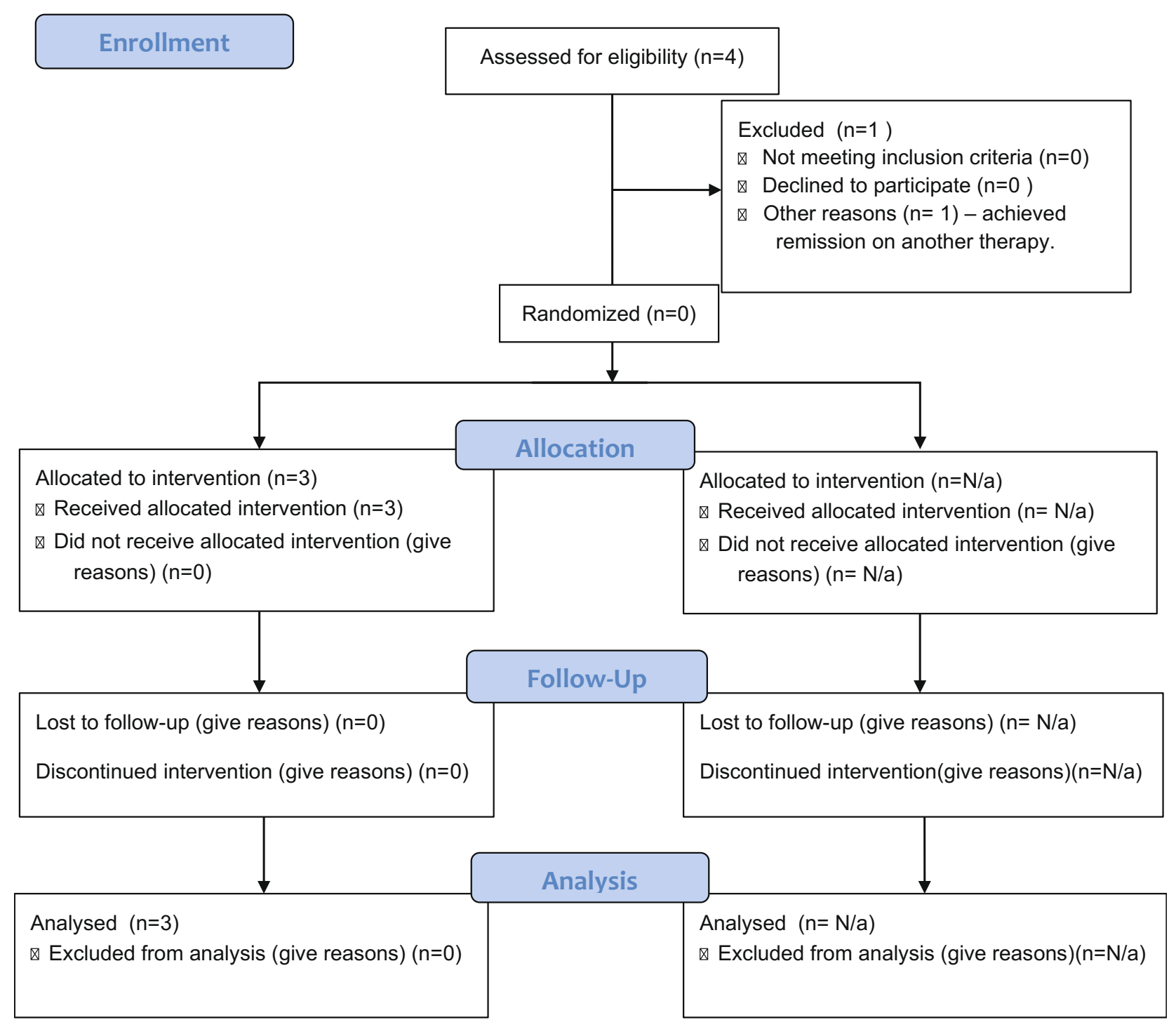

Extended Data Fig. 1 | Clinical trial design and enrollment. a, Trial design: Cyclophosphamide/fludarabine (Cy/Flu) chemotherapy is given on days -4 , -3 , and -2 (purple arrows). CAR-NKTs are infused on day 0 (green arrow). Tumor biopsy is performed at week 2 (blue arrow) and response evaluation with imaging at week 4 (range 4-6; orange arrow). Black arrows indicate clinic visits and peripheral blood analysis. b. Enrollment CONSORT flow diagram. 
Patient Characteristics

\begin{tabular}{|c|c|c|c|c|c|c|c|c|c|c|c|}
\hline $\bar{N}$ & $\begin{array}{l}\text { Age, } \\
\text { Yrs }\end{array}$ & Gender & INSS & $\begin{array}{l}\text { Involved } \\
\text { Sites }\end{array}$ & $\begin{array}{l}\text { Cyl } \\
\text { Flu }^{* *}\end{array}$ & $\begin{array}{c}\text { Dose } \\
\text { CAR+/total } \\
\text { NKT cells }\end{array}$ & Response & $\begin{array}{l}\text { Initial } \\
\text { Curie } \\
\text { Score }\end{array}$ & $\begin{array}{c}\text { Curie } \\
\text { Score } \\
\text { Week } 4\end{array}$ & $\begin{array}{l}\text { Long term } \\
\text { outcome }\end{array}$ & $\begin{array}{c}\text { Course after CAR } \\
\text { NKT infusion }\end{array}$ \\
\hline 1 & 12 & $\mathrm{M}$ & 4 & $\begin{array}{c}\text { Multifocal } \\
\text { bone and } \\
\text { bone } \\
\text { marrow, } \\
\text { soft tissues } \\
\text { and } \\
\text { paraspinal } \\
\text { masses }\end{array}$ & Yes & $\begin{array}{c}3 \times 10^{6} / \\
8.6 \times 10^{6}\end{array}$ & $\begin{array}{l}\text { Stable } \\
\text { Disease }\end{array}$ & 21 & 21 & DOD & $\begin{array}{l}\text { Received salvage } \\
\text { chemotherapy } \\
\text { without response. }\end{array}$ \\
\hline 2 & 12 & $\mathrm{M}$ & 4 & $\begin{array}{l}\text { Multifocal } \\
\text { bone }\end{array}$ & Yes & $\begin{array}{l}3 \times 10^{6} \% \\
1.5 \times 10^{7}\end{array}$ & $\begin{array}{c}\text { Partial } \\
\text { Response }\end{array}$ & 2 & 1 & AWD & $\begin{array}{l}\text { Received salvage } \\
\text { chemotherapy and } \\
\text { achieved CR for } \\
\text { approx. six months. }\end{array}$ \\
\hline 3 & 6 & $\mathrm{M}$ & 4 & $\begin{array}{l}\text { Multifocal } \\
\text { bone }\end{array}$ & Yes & $\begin{array}{l}3 \times 10^{6} \% \\
4.3 \times 10^{6}\end{array}$ & $\begin{array}{l}\text { Stable } \\
\text { Disease }\end{array}$ & 7 & 5 & AWD & $\begin{array}{l}\text { Received salvage } \\
\text { chemotherapy with } \\
\text { persistent disease. }\end{array}$ \\
\hline
\end{tabular}

Extended Data Fig. 2 | Patient characteristics. Indicated variables shown for the three patients enrolled on dose level 1. INSS: International Neuroblastoma Staging System; ${ }^{\star *}$ Cy: cyclophosphamide, Flu: fludarabine. \$: Dose: CAR-NKT cells / $\mathrm{m}^{2}$. Response criteria determined by revised International Neuroblastoma Response Criteria; partial response $=$ elimination of at least $50 \%$ of bone metastatic lesions. DOD: Died of disease. AWD: Alive with disease. 
a
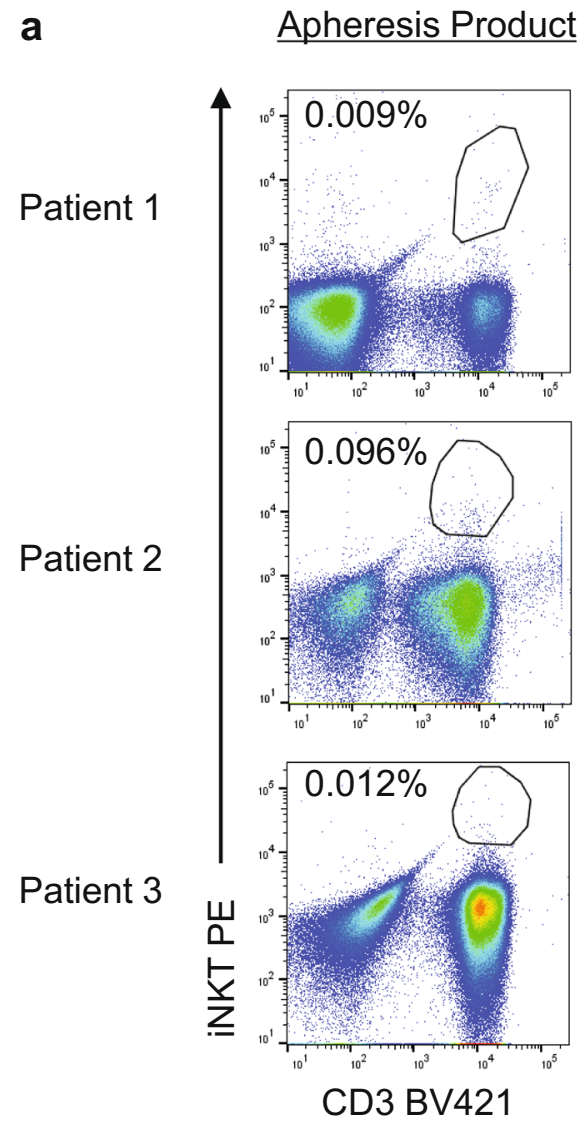

b

$\underline{\text { Final Product }}$

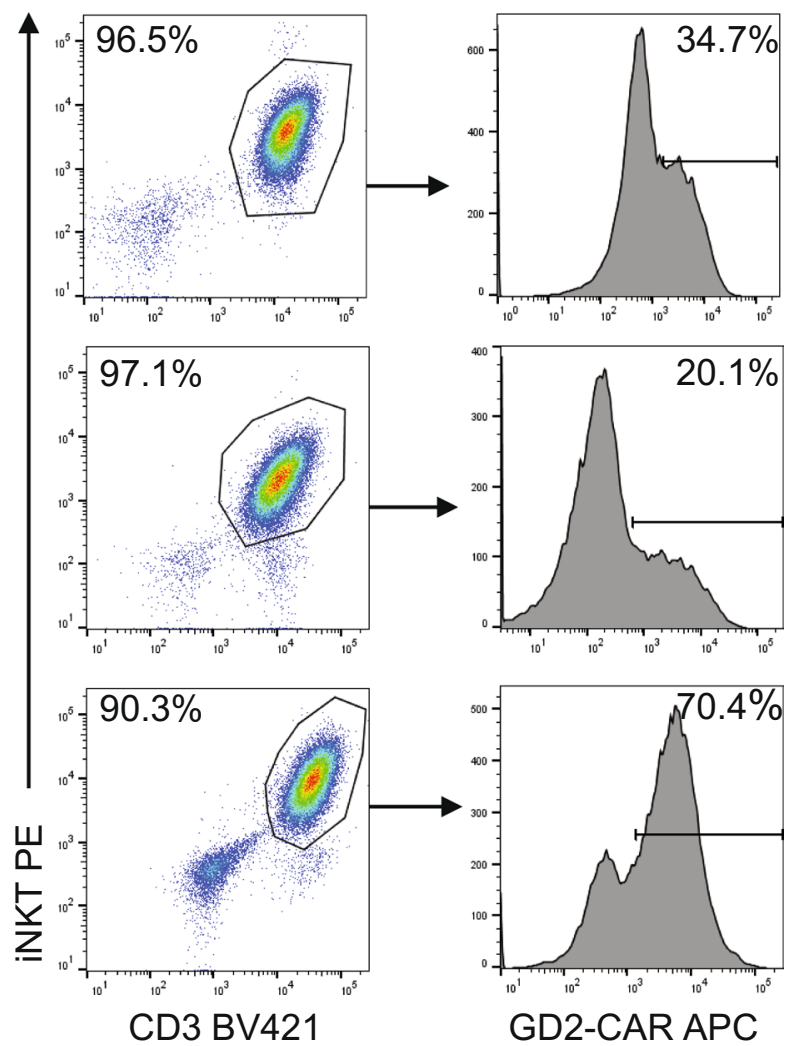

Extended Data Fig. 3 | Characteristics of GD2-CAR-NKT cell products. Patient cells were manufactured in the cGMP facility of the Center for Cell and Gene Therapy at Texas Children's Hospital and evaluated using multi-color flow cytometry. a, NKT frequency (CD3+iNKT+) in initial apheresis product for the three patients. b, NKT purity and CAR expression at the end of manufacturing prior to cryopreservation. Shown are results from one of two independent experiments that produced similar results. 


\begin{tabular}{|c|c|c|c|c|}
\hline \multirow{2}{*}{ Body System } & \multirow{2}{*}{ Event } & \multicolumn{3}{|c|}{ Grade } \\
\hline & & Patient 1 & Patient 2 & Patient 3 \\
\hline \multirow{5}{*}{$\begin{array}{l}\text { Blood and lymphatic system } \\
\text { disorders }\end{array}$} & Anemia & 3 & 2 & 1 \\
\hline & Lymphocyte count decreased & 4 & 4 & 3 \\
\hline & Neutrophil count decreased & 4 & 4 & 4 \\
\hline & Platelet count decreased & 4 & 1 & 1 \\
\hline & White blood cell decreased & 4 & 3 & 2 \\
\hline \multicolumn{2}{|c|}{ Metabolism and nutrition disorders Anorexia } & & 1 & \\
\hline & Dehydration & & 1 & \\
\hline & Hyperglycemia & & 1 & \\
\hline & Hypermagnesemia & & 1 & \\
\hline & Hyponatremia & 1 & & \\
\hline & AST increased & 1 & 1 & 1 \\
\hline $\begin{array}{l}\text { Musculoskeletal and connective } \\
\text { tissue disorders }\end{array}$ & Back pain & 1 & & \\
\hline $\begin{array}{l}\text { Respiratory, thoracic and } \\
\text { mediastinal disorders }\end{array}$ & Epistaxis & & 1 & \\
\hline
\end{tabular}

Extended Data Fig. 4 | Adverse events. Patients were monitored for adverse events from the beginning of lymphodepletion (day -4) until day 28 (four weeks post-infusion). Adverse events were described using Common Terminology Criteria for Adverse Events version 4. 
a
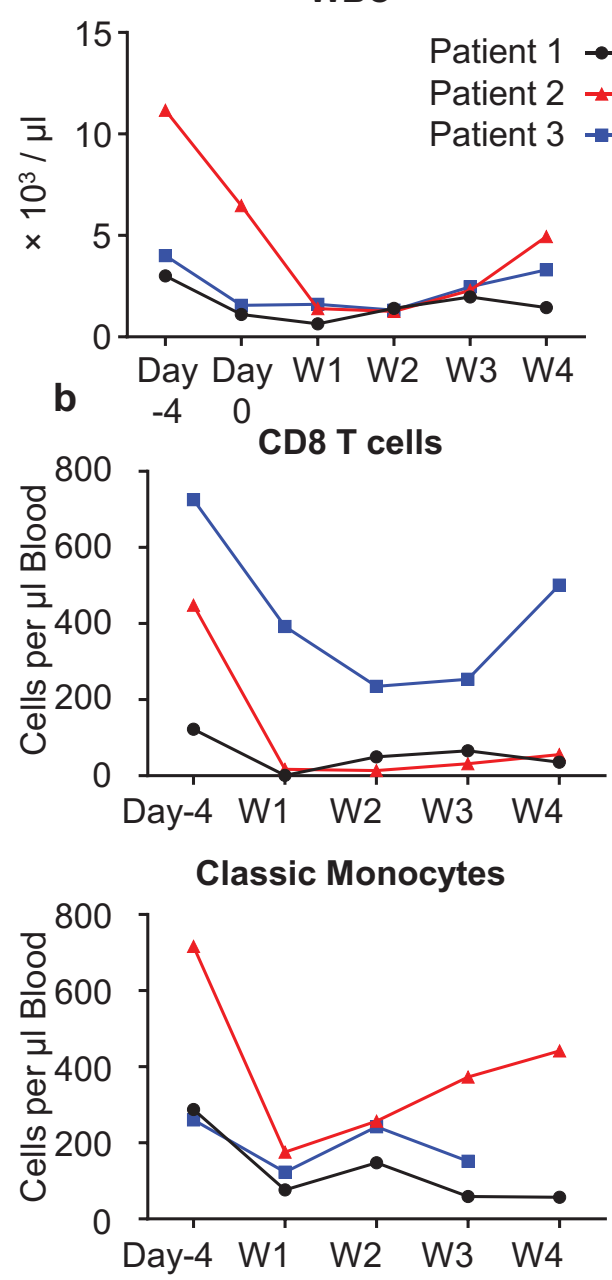

M-MDSC

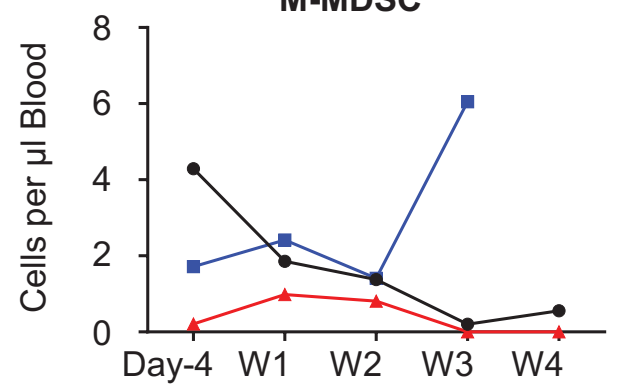

ALC
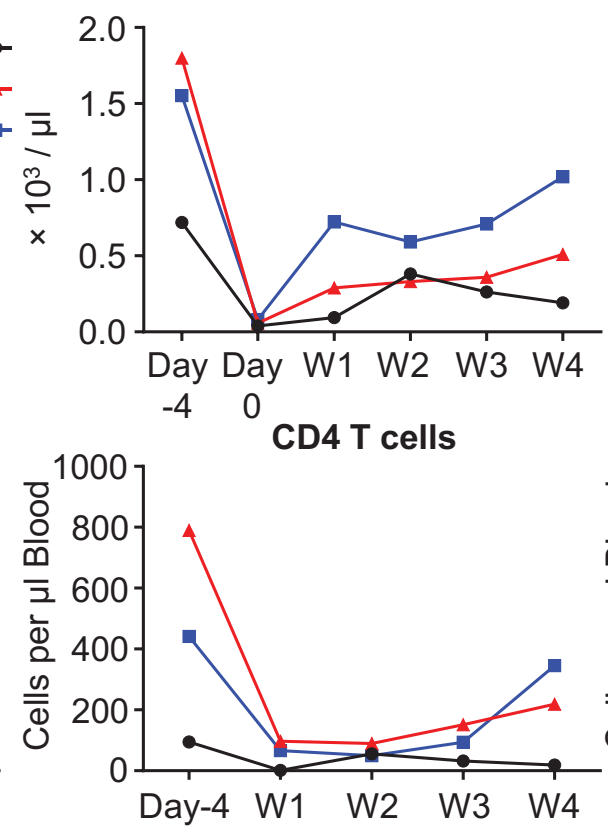

Non-classical monocytes

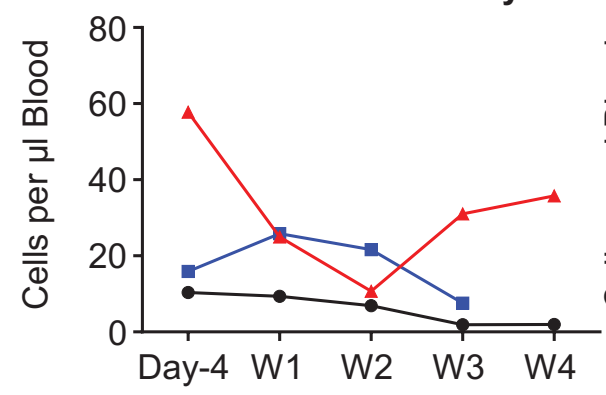

PMN-MDSC

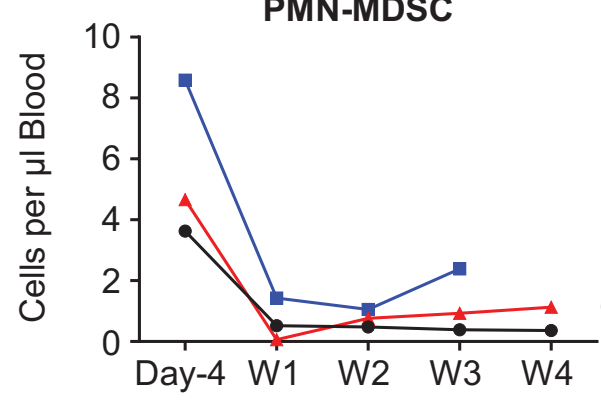

AMC
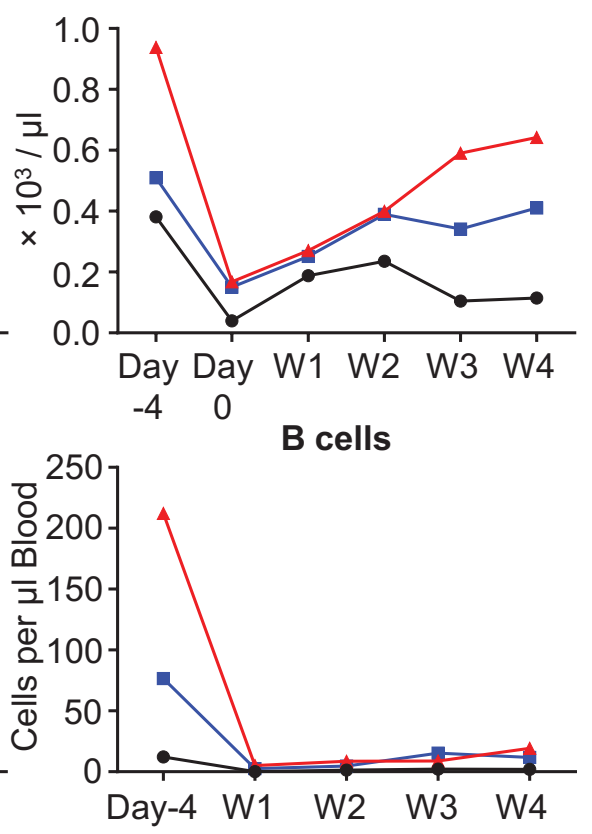

NK cells

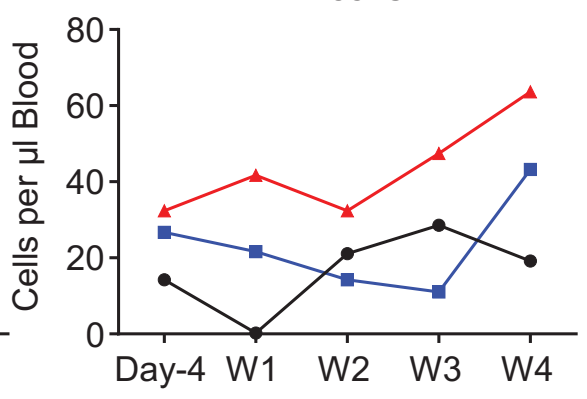

Tregs

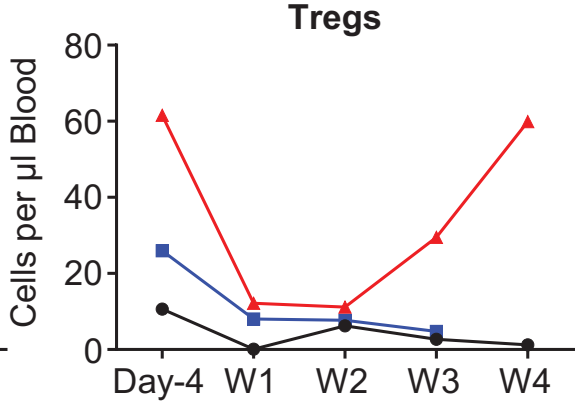

Extended Data Fig. 5 | Peripheral blood analysis pre- and post-infusion. Peripheral blood samples were collected at indicated timepoints and a) the frequency of total white blood cells (WBCs), absolute lymphocyte counts ( $A L C$ ), and absolute monocyte counts (AMC) were quantified in a standard clinical laboratory at the Texas Children's Cancer Center. b. Frequency of myeloid and lymphoid cell subsets were quantified by flow cytometry staining. Absolute numbers of these subsets in peripheral blood were determined using flow cytometry data and AMC or ALC, respectively. Live PBMCs were gated and cell subsets were defined as shown in Supplementary Fig. 5. For patient 3, analyses of Treg and monocyte subsets were not performed at the week 4 timepoint. 


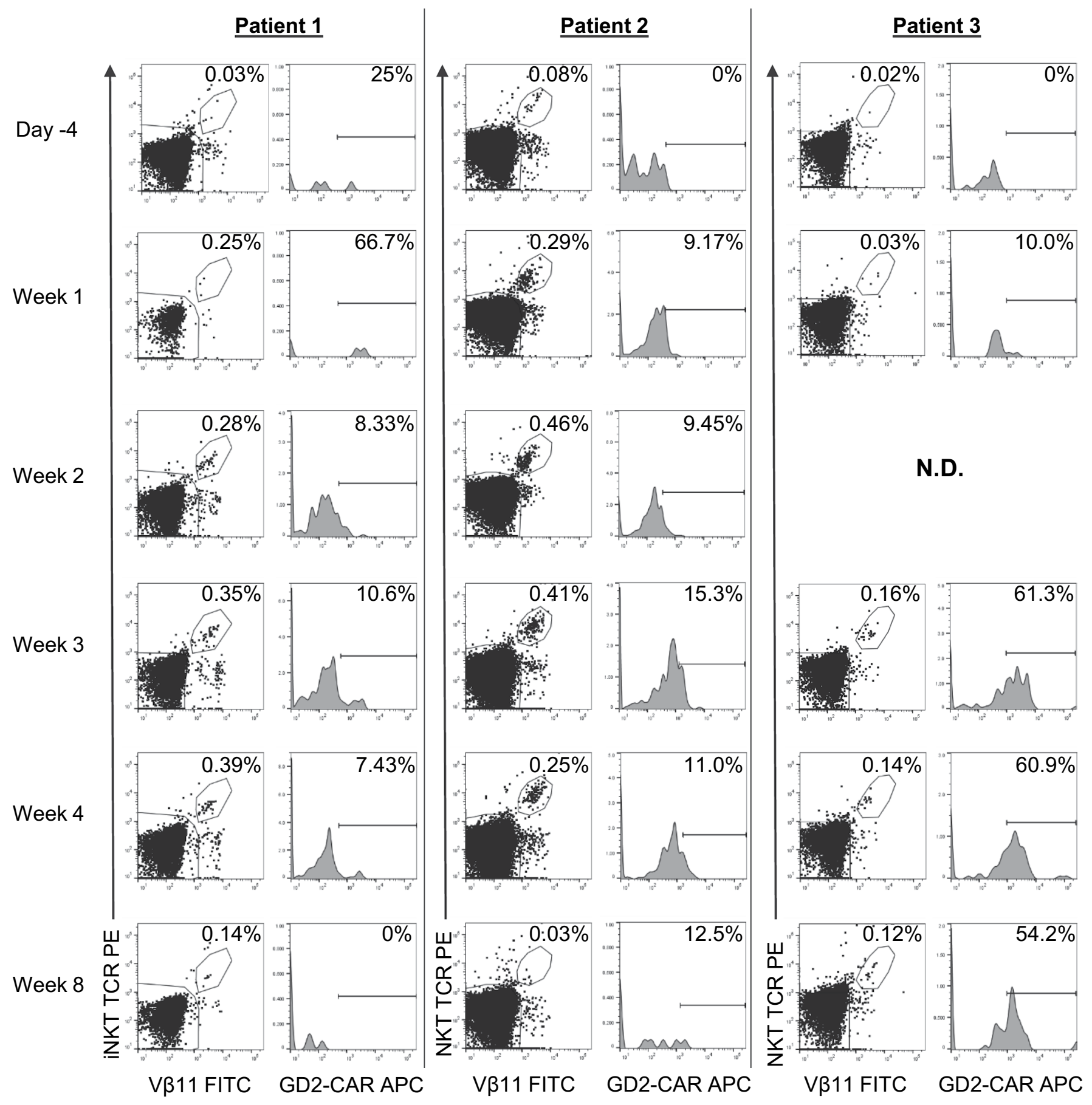

Extended Data Fig. 6 | Detection of CAR-NKT cells in treated patients pre- and post-infusion. Flow cytometry staining showing iNKT+V $\beta 11+$ cells after gating on CD3+ cells and corresponding GD2-CAR expression in patients at indicated timepoints. T-cells served as a negative control for CAR staining. N.D.: not defined; indicates that assay was not performed at given timepoint. 

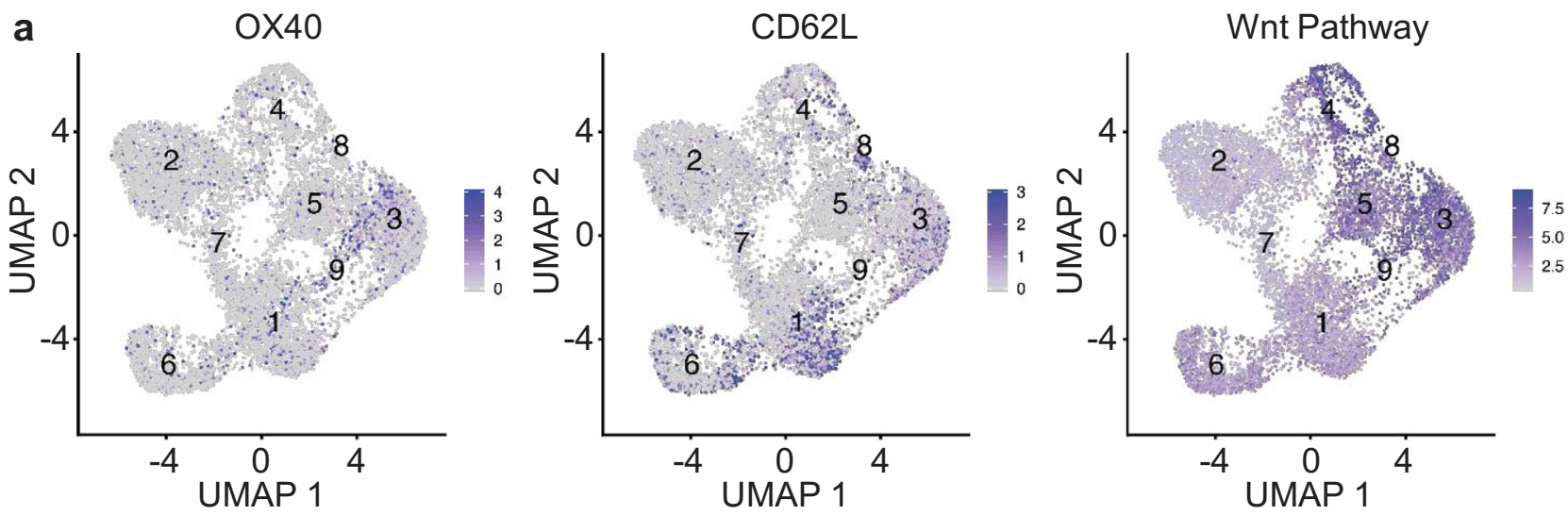

b Patient $1 \quad$ Patient $2 \quad$ Patient 3

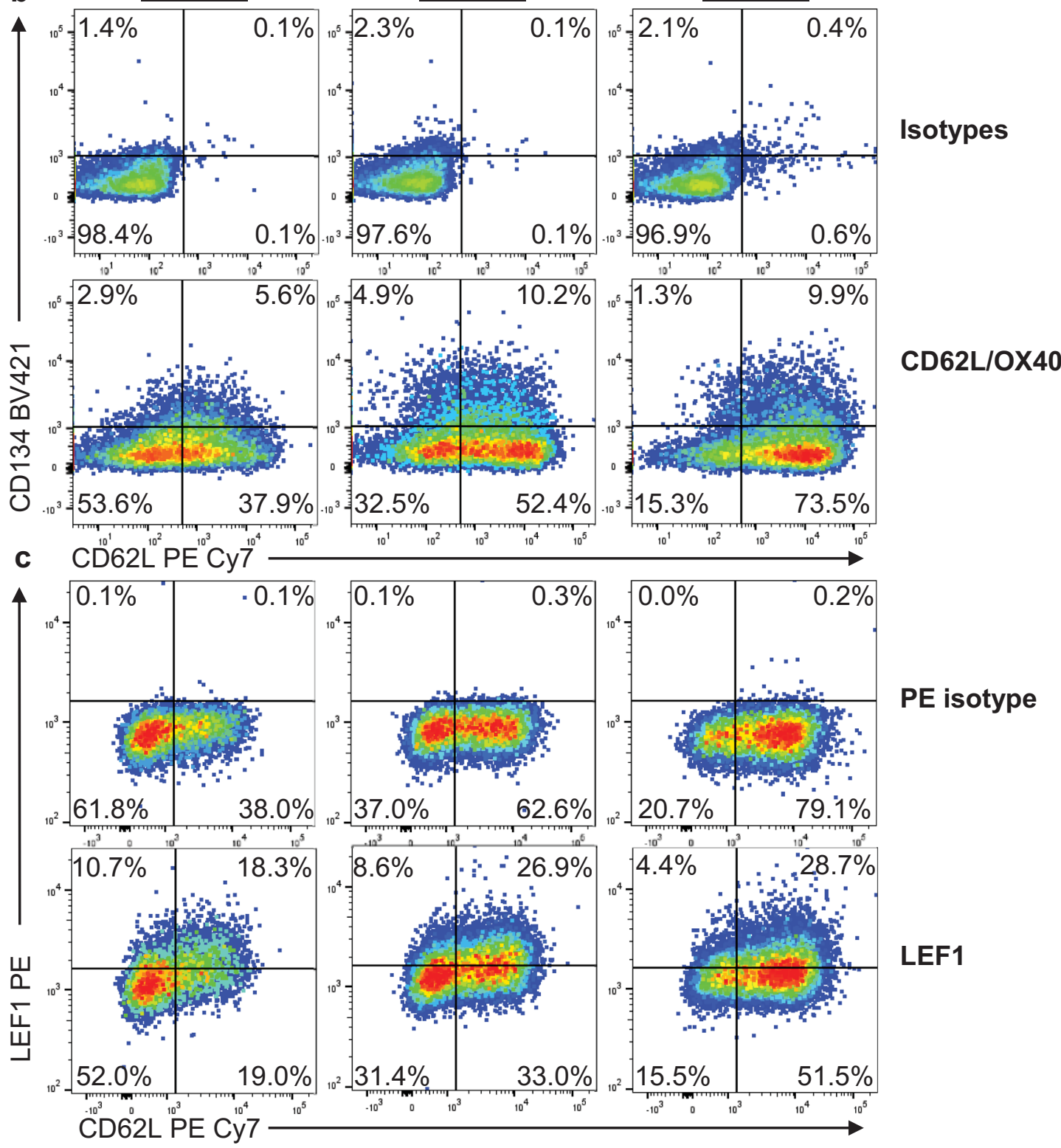

Extended Data Fig. 7 | Expression of central memory and WNT pathway genes in pre-infusion patient products. a, Gene expression in pre-infusion products from patients 1-3 for TNFRSF4 (encodes OX40), SELL (encodes CD62L), and genes related to Wnt-mediated signal transduction (https:// www.gsea-msigdb.org/gsea/msigdb/cards/WNT_SIGNALING.html) overlaid on the UMAP 2D projection. b, Cryopreserved patient products were thawed and rested overnight prior to evaluating surface expression of OX40 (CD134) and CD62L (bottom) by flow cytometry using the corresponding fluorochrome-conjugated isotype controls (top). c, LEF1 expression in relation to CD62L was analyzed using intracellular flow cytometry (bottom) with the corresponding PE-conjugated isotype controls (top). Results are from one of two independent experiments with similar results. 
a

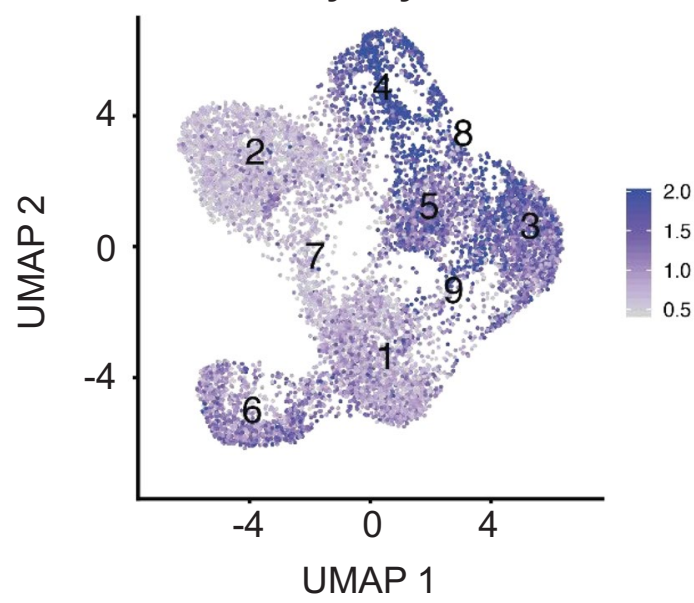

b

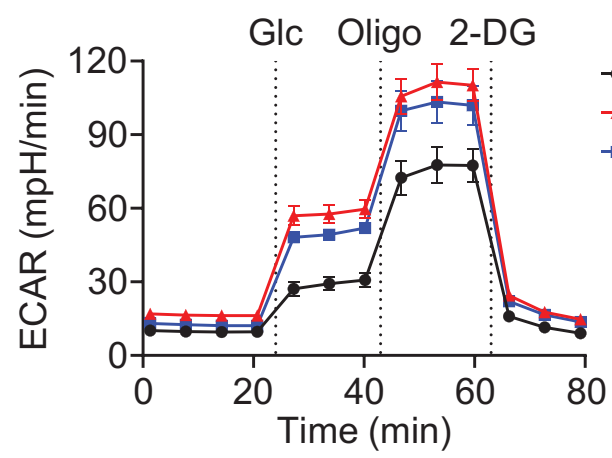

C Mitochondrial Respiration

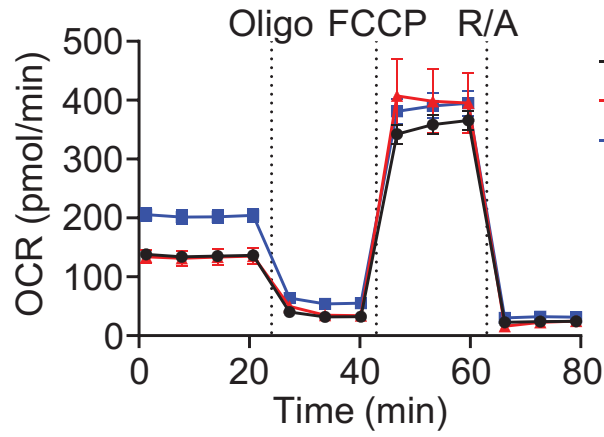

Oxidative Phosphorylation

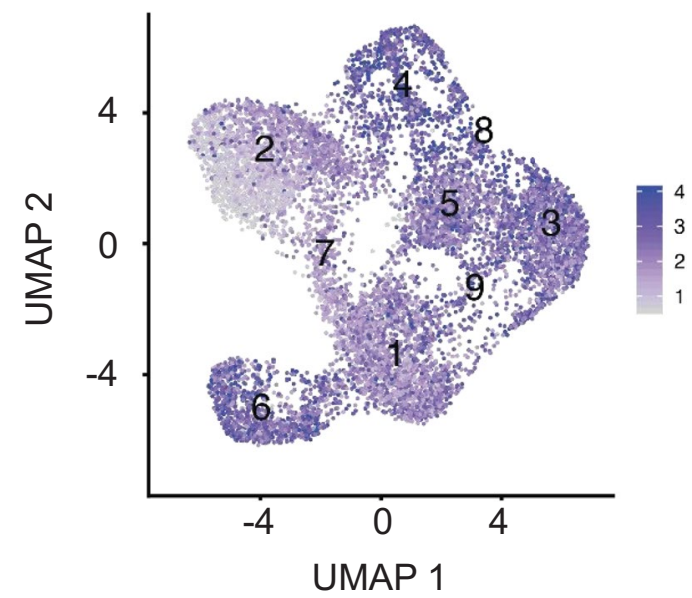

d

Glycolytic parameters

마ient 1

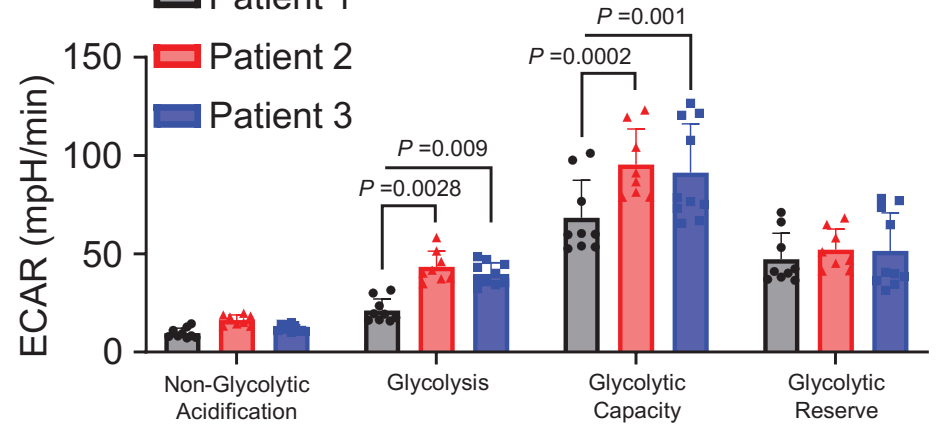

e Mitochondrial parameters

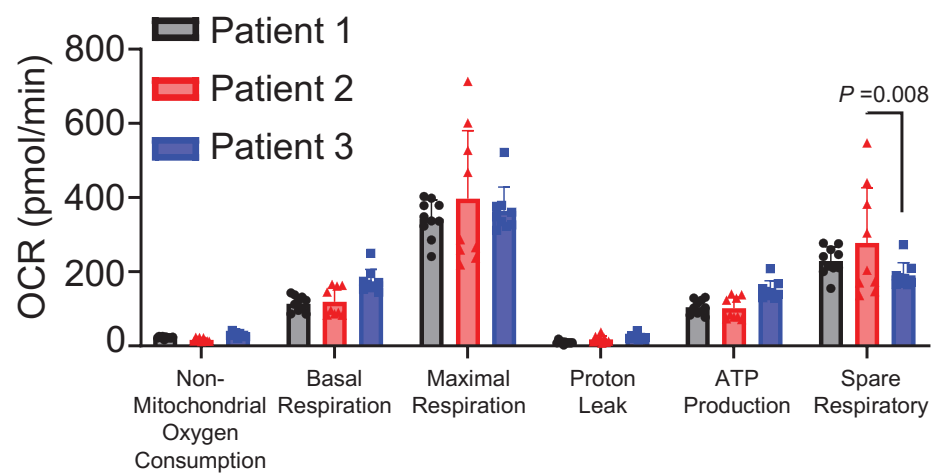

Extended Data Fig. 8 | Expression of metabolic genes and bioenergetic analysis of pre-infusion patient products. a, Gene expression in pre-infusion products from patients 1-3 related to glycolysis (https://www.gsea-msigdb.org/gsea/msigdb/cards/KEGG_GLYCOLYSIS_GLUCONEOGENESIS) and oxidative phosphorylation (https://www.gsea-msigdb.org/gsea/msigdb/cards/KEGG_OXIDATIVE_PHOSPHORYLATION) overlaid on the UMAP 2D projection. b. Extracellular acidification rates (ECAR) and $\mathbf{c}$ ) oxygen consumption rates (OCR) measured at baseline and in response to glucose (Glc), oligomycin (Oligo), 2-deoxy-D-glucose (2-DG), FCCP, or rotenone/antimycin A (R/A); data shown as mean \pm SEM. $\mathbf{d}$, Glycolytic and e) mitochondrial parameters derived from assay in $\mathbf{b}$ ) and $\mathbf{c}$ ) respectively; data shown as mean \pm SD; $P$ values by two-way ANOVA with Tukey's correction for multiple comparisons $(\mathbf{d}, \mathbf{e})$. Shown are results from two independent experiments with 3 - 6 replicates per experiment. 
a

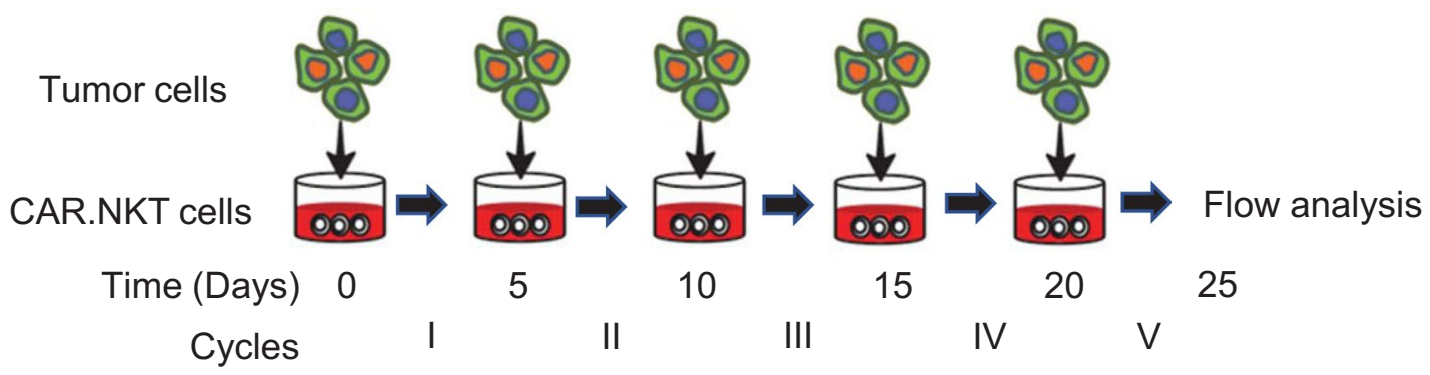

b

Patient 1

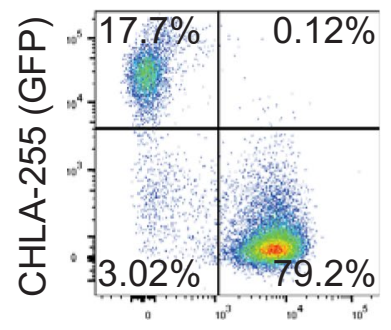

CD3 PE Cy7
Patient 2
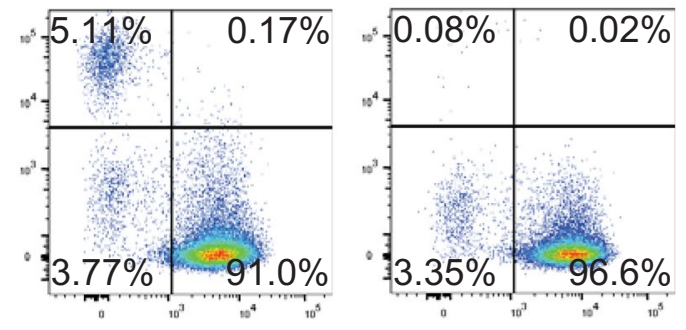

Patient 3

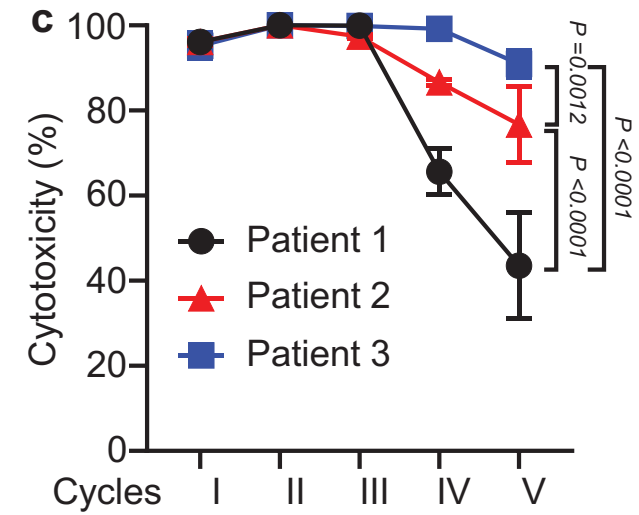

d

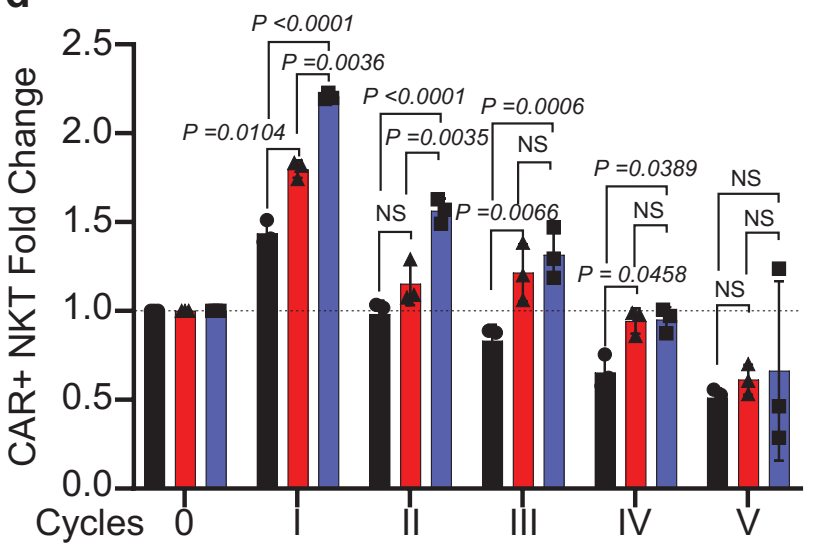

Patient \#1
Patient \#2
Patient \#3

Extended Data Fig. 9 | Serial tumor challenge assay. a, Patient GD2-CAR NKTs and NB cell line CHLA-255 were co-cultured in a 24-well plate. Five days later, cells were harvested for quantification by trypan-blue exclusion and FACS analysis. NKT cells were then re-plated at a 1:1 effector-to-target ratio with fresh tumor cells in fresh cell culture medium to start the next round with five rounds total. $\mathbf{b}$, Representative flow cytometry analysis after round 4. Summary of $\mathbf{c}$ ) tumor cell cytotoxicity and $\mathbf{d}$ ) GD2-CAR NKT proliferation at the end of each five-day cycle. Data shown as mean \pm SD from three technical replicates; $P$ values by two-way ANOVA with Tukey's correction for multiple comparisons (c and $\mathbf{d}$ ). 
a

Patient 1

Patient 2

Patient 3
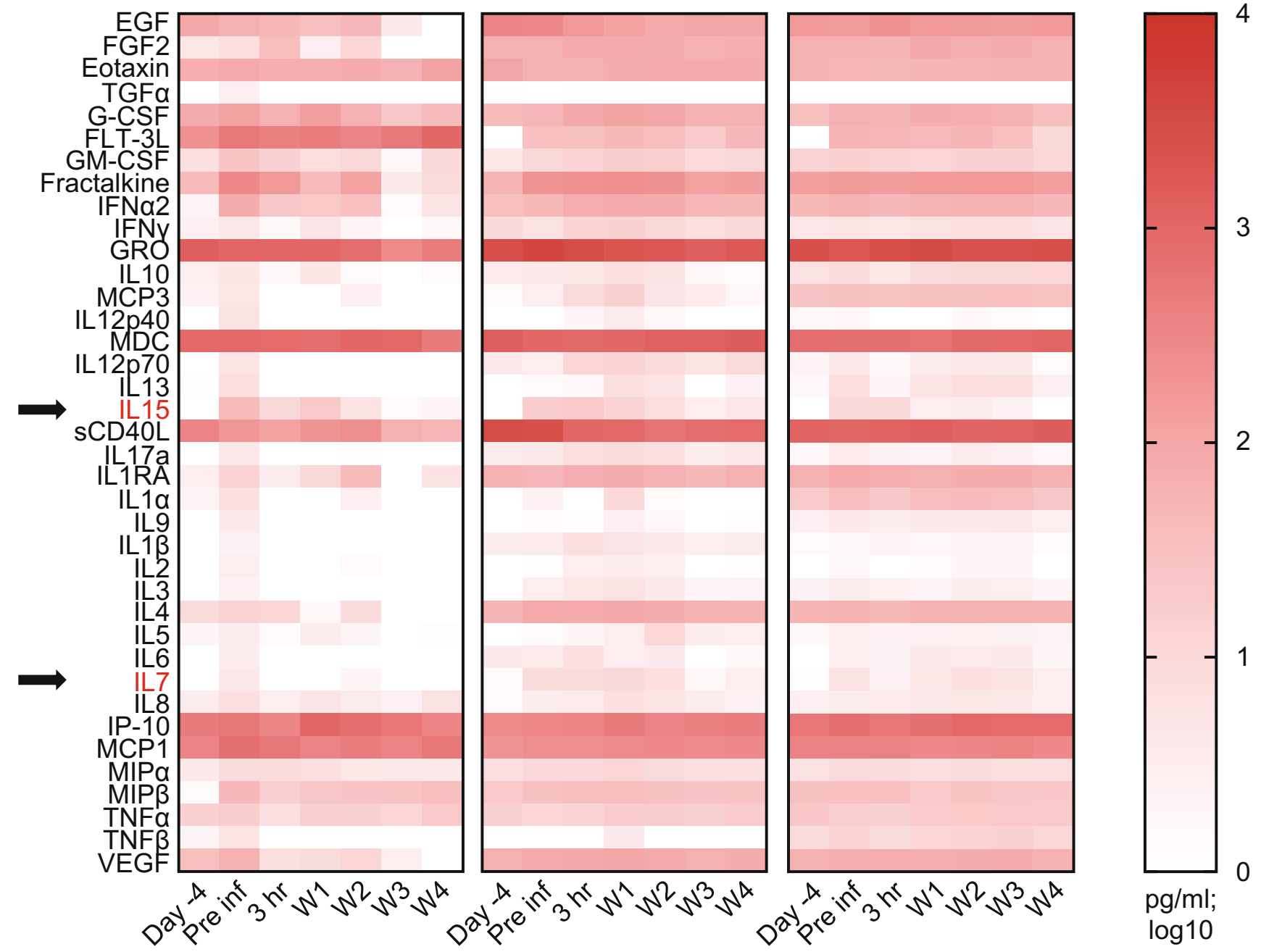

b

IL7

C

IL15

d

Peak IL15 concentration
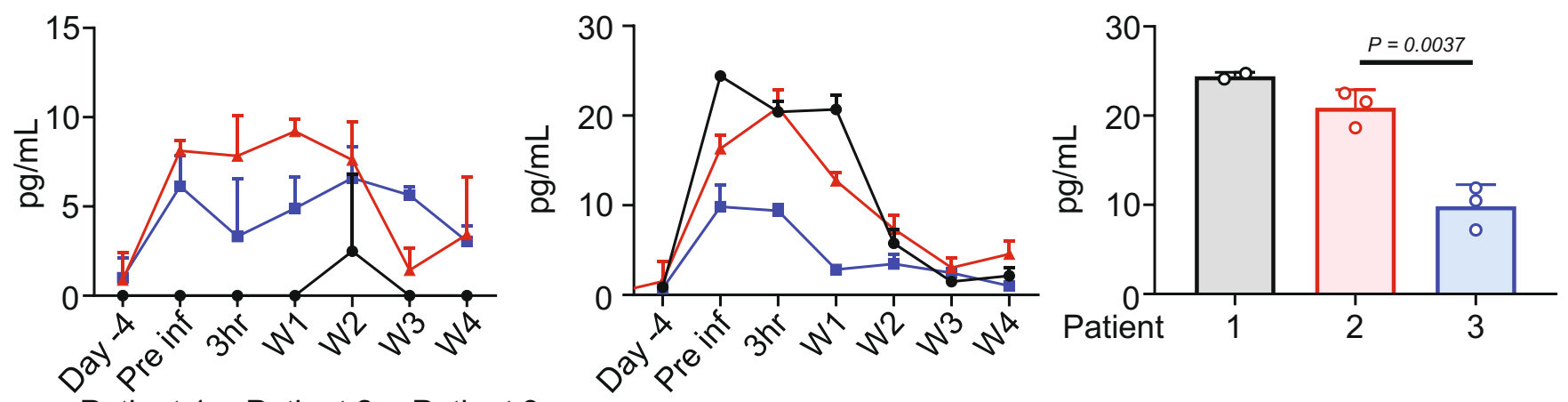

Extended Data Fig. 10 | Peripheral blood cytokine levels. Peripheral blood was collected at indicated timepoints from the three patients and cytokine levels were quantified in triplicate by Luminex ${ }^{\circledR}$ assay. a, Cytokine expression is shown as log 10 of pg/mL. b, IL7 and c) IL15 levels for the three patients at indicated timepoints. d, Peak IL15 concentration. Data shown as mean \pm SD from three technical replicates (b-d); $P$ values by unpaired two-tailed t-test (d). 